
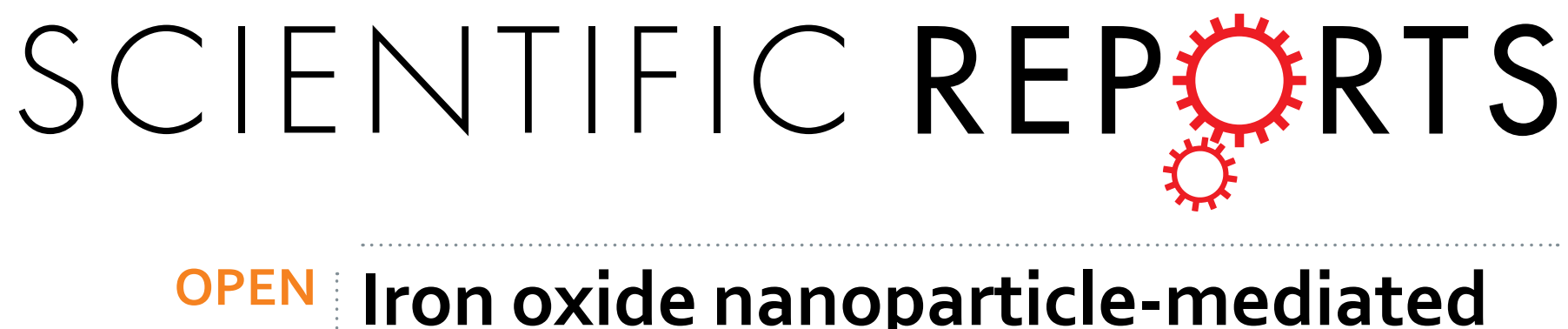

\title{
Iron oxide nanoparticle-mediated hyperthermia stimulates dispersal in bacterial biofilms and enhances \\ antibiotic efficacy
}

Received: 10 September 2015

Accepted: 17 November 2015

Published: 18 December 2015

\author{
Thuy-Khanh Nguyen ${ }^{1}$, Hien T. T. Duong ${ }^{1,{ }^{\dagger}}$, Ramona Selvanayagam ${ }^{1}$, Cyrille Boyer $^{1, *}$ \\ \& Nicolas Barraud ${ }^{2, *}, *$
}

The dispersal phase that completes the biofilm lifecycle is of particular interest for its potential to remove recalcitrant, antimicrobial tolerant biofilm infections. Here we found that temperature is a cue for biofilm dispersal and a rise by $5^{\circ} \mathrm{C}$ or more can induce the detachment of Pseudomonas aeruginosa biofilms. Temperature upshifts were found to decrease biofilm biomass and increase the number of viable freely suspended cells. The dispersal response appeared to involve the secondary messenger cyclic di-GMP, which is central to a genetic network governing motile to sessile transitions in bacteria. Furthermore, we used poly((oligo(ethylene glycol) methyl ether acrylate)-block-poly(monoacryloxy ethyl phosphate)-stabilized iron oxide nanoparticles (POEGA-b-PMAEP@IONPs) to induce local hyperthermia in established biofilms upon exposure to a magnetic field. POEGA-b-PMAEP@IONPs were non-toxic to bacteria and when heated induced the detachment of biofilm cells. Finally, combined treatments of POEGA- $b$-PMAEP@IONPs and the antibiotic gentamicin reduced by 2-log the number of colony-forming units in both biofilm and planktonic phases after $20 \mathrm{~min}$, which represent a 3.2- and 4.1fold increase in the efficacy against planktonic and biofilm cells, respectively, compared to gentamicin alone. The use of iron oxide nanoparticles to disperse biofilms may find broad applications across a range of clinical and industrial settings.

Bacteria have an extraordinary ability for adaptation, activating defence systems when put under stress, while thriving when the conditions improve. This capacity is often the cause of failed antibiotic and antimicrobial therapies with dramatic consequences both in healthcare and industrial contexts ${ }^{1}$. The study of bacterial adaptive behaviours and the molecular mechanisms that regulate them is crucial to develop novel effective antimicrobial strategies to treat infectious diseases and control contaminations.

In nature bacteria predominantly live in biofilms, which are sessile multicellular communities comprised of bacterial cells encased in a matrix of secreted polymeric substances. The biofilm mode of growth confers bacteria with increased resistance and persistence to external stress. If a biofilm successfully forms on host tissues, it often leads to chronic infections that antimicrobial treatments, which have been traditionally designed against planktonic bacteria, as well as immune defences fail to fully eradicate ${ }^{2}$. Biofilms have been linked to $80 \%$ of infections, forming on living tissues (e.g. respiratory, gastrointestinal and urinary tracts, oral cavity, eyes, ears, wounds, heart, cervix) or indwelling medical devices (e.g. dialysis catheters, prosthetic implants, contact lenses) ${ }^{3}$. In the industry, formation of biofilms can cause economic loss, for instance by inducing fouling and corrosion of wet surfaces or clogging of filtration membranes ${ }^{4}$. It can also pose a threat to public health by acting as reservoirs of pathogens in drinking water distribution systems ${ }^{5}$.

${ }^{1}$ Centre for Advanced Macromolecular Design (CAMD) and Australian Centre for NanoMedicine (ACN), School of Chemical Engineering, The University of New South Wales, Sydney 2052, Australia. ${ }^{2}$ Centre for Marine Bio-Innovation, School of Biotechnology and Biomolecular Sciences, The University of New South Wales, Sydney 2052, Australia. †Present address: School of Chemistry, The University of Sydney, Sydney 2006, Australia. ${ }^{\ddagger}$ Present address: Department of Microbiology, Genetics of Biofilms Unit, Institut Pasteur, Paris 75015, France. ${ }^{*}$ These authors contributed equally to this work. Correspondence and requests for materials should be addressed to C.B. (email: cboyer@unsw.edu.au) or N.B. (email: n.barraud@melix.org) 
The biofilm lifestyle appears to be the main phase of a biphasic lifecycle, of which the other phase is the single-celled, dispersive planktonic lifestyle. Bacteria have the ability to switch between these two states via transition stages of attachment or dispersal. While the attachment stage has been well documented in several species, the reverse transition of dispersal has been much less studied despite its interest for developing novel strategies to control and remove unwanted biofilms ${ }^{6}$. Dispersal, which consists in the coordinated release of single cells or small aggregates from the interior of mature biofilms, is a key adaptive strategy that allows bacterial communities to spread and colonize new surfaces ${ }^{7}$. Recent studies have shown that biofilm dispersal can be induced in response to a range of environmental cues including changes in the availability of nutrients such as carbon sources ${ }^{8,9}$ or oxygen $\left(\mathrm{O}_{2}\right)^{10}$, low levels of nitric oxide $(\mathrm{NO})^{11}$ or iron levels ${ }^{12}$, as well as several bacterially derived signals including quorum sensing (QS) acyl-homoserine lactones ${ }^{13}$, autoinducing peptides ${ }^{14}$ and diffusible fatty acids ${ }^{15,16}$. Furthermore, the regulation of biofilm dispersal involves intracellular secondary messengers such as nucleotides cyclic di-GMP (c-di-GMP) and cyclic AMP ${ }^{7}$. c-di-GMP is the central element of a complex regulatory network that also includes sensor and effector proteins and controls the transition between planktonic and biofilm lifestyles ${ }^{17}$. Via this network, environmental cues and signals can stimulate enzymes that modify intracellular levels of c-di-GMP, which in turn regulates a range of downstream effectors leading to biofilm formation or dispersal. Diguanylate cyclases (DGC) that synthesize c-di-GMP typically increase biofilm formation when high intracellular levels of c-di-GMP induce the production of extracellular matrix polysaccharides or fimbriae adhesins. Conversely, phosphodiesterases (PDE) that degrade c-di-GMP favour a motile planktonic lifestyle by inducing the degradation of the biofilm matrix components and expression of flagella and chemotaxis genes ${ }^{17}$. Thus biofilm dispersal appears to be an active, highly regulated process responsive to changes in environmental conditions.

The role of temperature variation, a common cue for life cycle transitions in eukaryotic ecology ${ }^{18}$, has received little attention in biofilm studies so far. Several reports suggest that it may be involved in regulating biofilm to planktonic transitions. Early work showed that oral bacteria such as Neisseria subflava, Haemophilus and Streptococcus species exhibit colony dispersal phenotypes in response to temperature gradients ${ }^{19}$. In Yersinia pestis, the pathogen responsible for the plague disease, RNA transcripts encoding for a DGC were found to be degraded at temperatures above $34^{\circ} \mathrm{C}$, thus repressing the production of biofilm-promoting factors ${ }^{20}$. A link between temperature change and c-di-GMP was also established in Escherichia coli. A PDE containing a blue light-sensing BLUF domain was found to dimerise and be inactive at low temperatures $\left(\sim 20^{\circ} \mathrm{C}\right)$ while the active monomer form that degrades c-di-GMP was stable at higher temperatures $\left(37^{\circ} \mathrm{C}\right)^{21}$. Finally, temperature shifts were recently shown to affect biofilm formation in the pathogen Vibrio cholerae. Decreases in temperature from $37^{\circ} \mathrm{C}$ to $25^{\circ} \mathrm{C}$ or $15^{\circ} \mathrm{C}$ were found to trigger an increase in intracellular levels of c-di-GMP and increased biofilm biomass, and a mutant lacking 6 DGC-encoding genes did not form biofilms in response to a temperature downshift ${ }^{22}$

In this study, we first explored the effect of temperature variations on biofilms of the opportunistic human pathogen and model organism Pseudomonas aeruginosa. We found that increases in temperature can induce the dispersal of pre-established biofilms while temperature decreases resulted in less cells shed from the biofilm. Based on these observations, we then assessed the potential of using iron oxide nanoparticles (IONPs) to induce local hyperthermia and disperse biofilms. IONPs are inorganic nanomaterials with unique physical properties including high surface area to volume ratios and superparamagnetism, which allows them to absorb the energy of an alternating magnetic field and convert it into heat. IONPs have good safety profiles and are commonly used as contrast agents during magnetic resonance imaging (MRI), as well as being investigated for their potential to increase tissue temperature and kill tumour cells by hyperthermia ${ }^{23}$. Due to their high surface energies and capacity to adsorb proteins, IONPs have a tendency to rapidly form aggregates in biological media, which can limit their usage in vivo. Coating the IONPs surface with polymers has been shown to stabilize and maintain the nanoparticles in a dispersed state, as well as allowing for addition of multiple functionalities such as conjugation with therapeutic (e.g. antibiotic) or targeting (e.g. antibody) agents and permeation enhancers ${ }^{24,25}$. Herein we report the synthesis of block copolymer poly((oligo(ethylene glycol) methyl ether acrylate)-block-poly(monoacryloxy ethyl phosphate)-coated IONPs (POEGA-b-PMAEP@IONPs) and demonstrate their efficacy at dispersing biofilms in a non-toxic manner while greatly improving the outcome of antibiotic therapies.

\section{Results}

Temperature upshifts induce biofilm dispersal in $P$. aeruginosa. The effect of temperature changes on biofilm dispersal was first assessed by using a continuous flow microfermentor culture system ${ }^{26}$. P. aeruginosa biofilms were grown for $24 \mathrm{~h}$ at $25^{\circ} \mathrm{C}, 30^{\circ} \mathrm{C}$ or $37^{\circ} \mathrm{C}$ before the temperature was suddenly changed. In all cases, increasing the temperature by $\geq 5^{\circ} \mathrm{C}$ resulted in the rapid release of dispersal cells in the biofilm effluent, as seen by increases in $\mathrm{OD}_{600}$ measurement. A shift by $8^{\circ} \mathrm{C}$ from $37^{\circ} \mathrm{C}$ to $45^{\circ} \mathrm{C}$ gave the strongest dispersal response with a peak showing a 3.3-fold increase in dispersed cells biomass compared to basal detachment levels (Fig. 1A). A shift from $30^{\circ} \mathrm{C}$ to $37^{\circ} \mathrm{C}$ and $25^{\circ} \mathrm{C}$ to $37^{\circ} \mathrm{C}$ resulted in 2.0 -fold increase in dispersal cells, while a shift from $25^{\circ} \mathrm{C}$ to $30^{\circ} \mathrm{C}$ only resulted in $10 \%$ increase in dispersal. Microscopy analysis of the biofilm effluent confirmed that increases in $\mathrm{OD}_{600}$ in the biofilm effluent correlated with an increase in the number of bacterial cells (data not shown). In contrast, when the temperature was reduced from $30^{\circ} \mathrm{C}$ to $25^{\circ} \mathrm{C}$ or from $37^{\circ} \mathrm{C}$ to $25^{\circ} \mathrm{C}$ or $15^{\circ} \mathrm{C}$, the amount of biomass released in the effluent appeared to decrease as seen by a reduction of $45 \%$ to $55 \%$ in $\mathrm{OD}_{600}$ measurements, suggesting that basal, non-specific cell detachment was inhibited upon temperature downshift (Fig. 1A). Taken together these data strongly suggest that hyperthermia associated with an increase in temperature is a potent signal for biofilm dispersal.

Because the intracellular messenger c-di-GMP is a master regulator of motile to sessile transitions in bacteria and was already found to be linked to temperature sensing in bacteria ${ }^{21,22}$, we then assessed its potential role in regulating temperature-mediated biofilm dispersal. A mutant strain carrying the arabinose-inducible expression vector $\mathrm{pJN} 105-w s p R$ (iDGC) was grown in microfermentors and expression of the DGC WspR was induced by adding arabinose $1 \mathrm{~h}$ before shifting the temperature. This timing allowed sufficient time to increase intracellular 

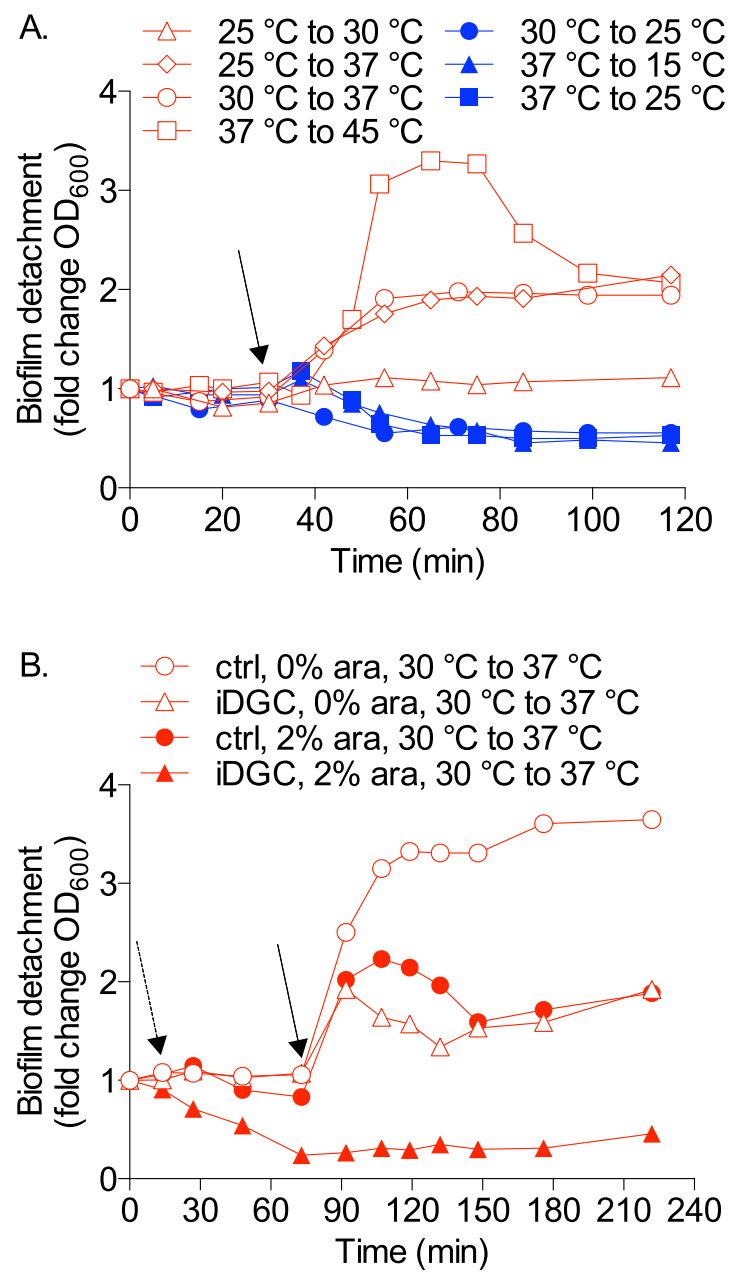

Figure 1. Temperature upshifts induce biofilm dispersal. (A) P. aeruginosa biofilms were grown in continuous flow microfermentor cultures at $25^{\circ} \mathrm{C}, 30^{\circ} \mathrm{C}$ or $37^{\circ} \mathrm{C}$ for $24 \mathrm{~h}$, before switching the temperature. The biofilm effluent was collected at regular intervals and quantified at $\mathrm{OD}_{600}$. (B) P. aeruginosa strains carrying the empty vector pJN105 (ctrl) or pJN105-wspR (iDGC) which allows for the arabinose-inducible expression of the DGC WspR were grown in microfermentors for $24 \mathrm{~h}$ at $30^{\circ} \mathrm{C}$ without arabinose. Then, the biofilms were treated with $2 \%$ arabinose (ara) or left untreated for $1 \mathrm{~h}$ before increasing the temperature. Solid arrows indicate temperature upshift or downshift, while dashed arrow indicates addition of arabinose. The data shown are representatives from at least $2(\mathbf{A}), 25^{\circ} \mathrm{C}$ to $30^{\circ} \mathrm{C}, 37^{\circ} \mathrm{C}$ to $45^{\circ} \mathrm{C}$ and temperature decreases) or 3 (A), $25^{\circ} \mathrm{C}$ or $30^{\circ} \mathrm{C}$ to $37^{\circ} \mathrm{C}$; (B) independent replicate experiments.

levels of c-di-GMP from DGC activity. Under these conditions that maintained high c-di-GMP levels, the dispersal response to temperature upshift from $30^{\circ} \mathrm{C}$ to $37^{\circ} \mathrm{C}$ was completely inhibited (Fig. 1B). In the absence of arabinose induction, the iDGC mutant exhibited reduced dispersal compared to the control experiment, which may be explained by leaky activity of the $\mathrm{P}_{\text {bad }}$ promoter and expression of the DGC WspR in this strain. Addition of arabinose to the vector control strain also appeared to affect the dispersal response, a phenomenon which had been observed in a previous study in P. aeruginosa PA $14^{27}$. Next, the potential role of the c-di-GMP-responsive periplasmic protease LapG was assessed. In Pseudomonas putida and Pseudomonas fluorescens biofilms, starvation was found to trigger a decrease in intracellular c-di-GMP levels which in turn activated LapG to cleave adhesins and lead to surface detachment ${ }^{28,29}$. Here, biofilms of a $P$. aeruginosa transposon mutant unable to express lap $G$ were found to not disperse in response to a range of cues in microfermentors assays, namely $\mathrm{NO}$ signals, $\mathrm{O}_{2}$ depletion and starvation (Supplementary information, Fig. S1A), which are all associated with decreases in intracellular c-di-GMP $9,30,31$. These data confirm that the protease LapG plays a prominent role in the regulation of dispersal in $P$. aeruginosa. Surprisingly, the lap $G$ mutant strain was not fully inhibited in the dispersal response to temperature upshift from $30^{\circ} \mathrm{C}$ to $37^{\circ} \mathrm{C}$ in the microfermentor assay (Supplementary information, Fig. S1B) nor from $37^{\circ} \mathrm{C}$ to $45^{\circ} \mathrm{C}$ (data not shown), suggesting that biofilm cell detachment in this assay involves other mechanisms than LapG-mediated proteolysis.

Synthesis and thermal characterization of block copolymer POEGA-b-PMAEP-coated IONPs. The use of IONPs allows for local control of temperature and inducing hyperthermia. To induce local hyperthermia to biofilms, we made block copolymer POEGA- $b$-PMAEP-stabilized IONPs. First block copolymers 

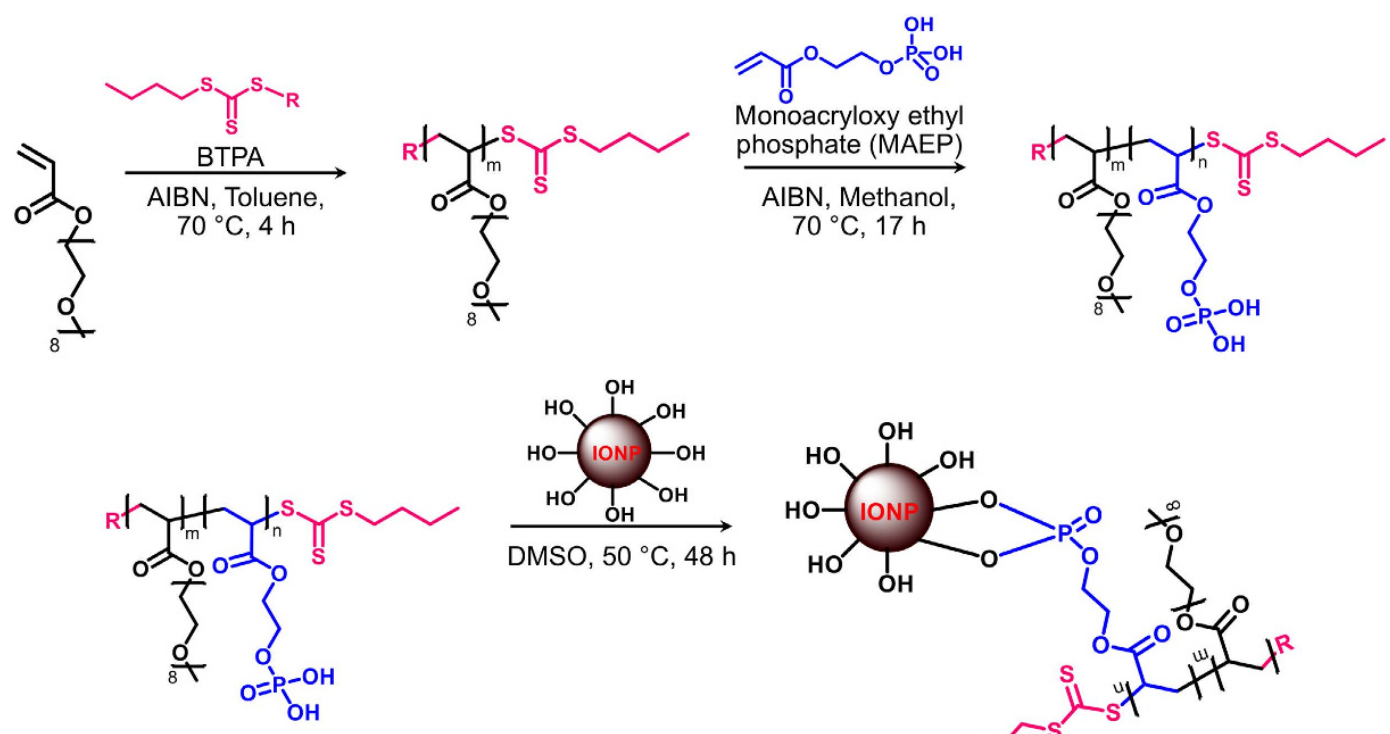

Figure 2. Schematic approach for the preparation of POEGA-b-PMAEP@IONPs via RAFT polymerization and "grafting onto" method.

POEGA- $b$-PMAEP were synthesized by using living radical polymerization (i.e. reversible addition fragmentation chain transfer (RAFT) polymerization) (Fig. 2). Poly((oligo(ethylene glycol) methyl ether acrylate) (POEGA) macro-RAFT agent was prepared in toluene at $70^{\circ} \mathrm{C}$ in the presence of $n$-butyltrithiocarbonate isopropionate (BTPA) as a RAFT agent and oligo(ethylene glycol) methyl ether acrylate (OEGA) as monomer. The conversion of the monomer was determined using ${ }^{1} \mathrm{H}$ NMR spectroscopy by comparing the intensity of vinyl proton peaks (6.5 and $5.9 \mathrm{ppm}$ ) to that of ester $-\mathrm{OCH}_{2}$ proton peaks $(\delta \sim 4.1 \mathrm{ppm})$. At $\sim 80 \%$ monomer conversion, the polymerization was stopped to avoid the formation of significant dead polymers, and the polymer was purified by several precipitations in a mixture of petroleum ether and diethyl ether $(70: 30, \mathrm{v} / \mathrm{v})$. The molecular weight of POEGA obtained by SEC analysis was in agreement with the theoretical value $\left(M_{n(t h e o)}=10,000 \mathrm{~g} \mathrm{~mol}^{-1}, M_{n(S E C)}=9,400 \mathrm{~g} \mathrm{~mol}^{-1}\right.$, polydispersity index $(\mathrm{PDI})=1.15)$. POEGA was successfully prepared with low molecular weight distribution and used as a macro-RAFT agent for the polymerization with monoacryloxy ethyl phosphate (MAEP) to afford block copolymer POEGA- $b$-PMAEP. The copolymers were dialyzed against methanol for $48 \mathrm{~h}$ to remove unreacted MAEP monomers. After purification, ${ }^{1} \mathrm{H}$ and ${ }^{31} \mathrm{P}$ NMR spectra confirmed MAEP incorporation by the presence of characteristic signals at $\sim 2.6 \mathrm{ppm}$ and $-0.15 \mathrm{ppm}$, respectively (Supplementary information, Fig. S1). The conversion of the MAEP was determined to be about $50 \%$ by using ${ }^{1} \mathrm{H}$ NMR spectroscopy and SEC $\left(\mathrm{M}_{n(\text { theo })}=11,000 \mathrm{~g} \mathrm{~mol}^{-1}\right.$, $\left.M_{n(S E C)}=11,200 \mathrm{~g} \mathrm{~mol}^{-1}, \mathrm{PDI}=1.18\right)$. SEC analysis confirmed that the chains successfully extended and that the molecular weight distribution shifted to higher molecular weight while the polydispersity remained low $(\mathrm{PDI}<1.20)$ (Supplementary information, Fig. S2).

Next, IONPs were prepared by the co-precipitation method ${ }^{32,33}$, yielding a mixture of magnetite $\mathrm{Fe}_{3} \mathrm{O}_{4}$ and maghemite $\gamma-\mathrm{Fe}_{2} \mathrm{O}_{3}$. Characterization by X-ray diffraction analysis (XRD) revealed a typical pattern corresponding to the spinel structure of $\gamma-\mathrm{Fe}_{2} \mathrm{O}_{3}$ and $\mathrm{Fe}_{3} \mathrm{O}_{4}$ (Fig. 3A). Then, IONPs were conjugated with the block copolymers. Dynamic light scattering (DLS) analysis revealed that the hydrodynamic diameter of IONPs increased from $16 \mathrm{~nm}$ to $44 \mathrm{~nm}$ upon polymer grafting (Fig. 3B). The morphology of bare IONPs and polymer-grafted IONPs was also analysed by transmission electron microscopy (TEM), which measures the dried form of the nanoparticles (Fig. 3C). The dried nanoparticles showed a uniformly distributed spherical shape, with a size of about $12 \mathrm{~nm}$, which did not appear to increase after polymer conjugation. Since TEM does not detect the POEGA- $b$-PMAEP polymers, this observation confirms that the increase in size observed by DLS analysis accounted for the successful polymer grafting rather than an increase in size of the IONPs. The combination of DLS and TEM analyses showed the good colloidal stability of the prepared IONPs for biomedical application. The polymer content in the POEGA-b-PMAEP@IONPs hybrids was then determined by thermogravimetric analysis (TGA), which measures the weight loss of polymers upon grafting onto metal nanoparticles indicative of the grafting density. A weight loss of about $34 \%$ was observed for the polymer-IONPs hybrids, corresponding to a grafting density of approximately 0.25 polymer chain per $\mathrm{nm}^{2}$ (Supplementary information, Fig. S3).

To characterize the thermal properties of IONP-conjugated polymers, bare IONPs or POEGA- $b$-PMAEP@ IONPs solutions $(1 \mathrm{~mL})$ in phosphate-buffered saline (PBS, $\mathrm{pH} 7.4$ ) were placed inside the coil of an alternating current (AC) magnetic field generator. Internal or external factors that prevent the IONPs magnetic dipole from following the applied alternating magnetic field result in the conversion of the magnetic energy into dissipated heat $^{34}$. Here we observed a IONP dose-dependent increase in temperature upon magnetic field activation (Fig. 4). Initially, the temperature went up rapidly before reaching a plateau after the first $10 \mathrm{~min}$, regardless of the IONPs concentration. A buffer only solution without any IONP that was used as a negative control, was found to warm up to $34^{\circ} \mathrm{C}$ due to heat radiating from the magnetic field generator coil. In the presence of a magnetic field bare 
A.
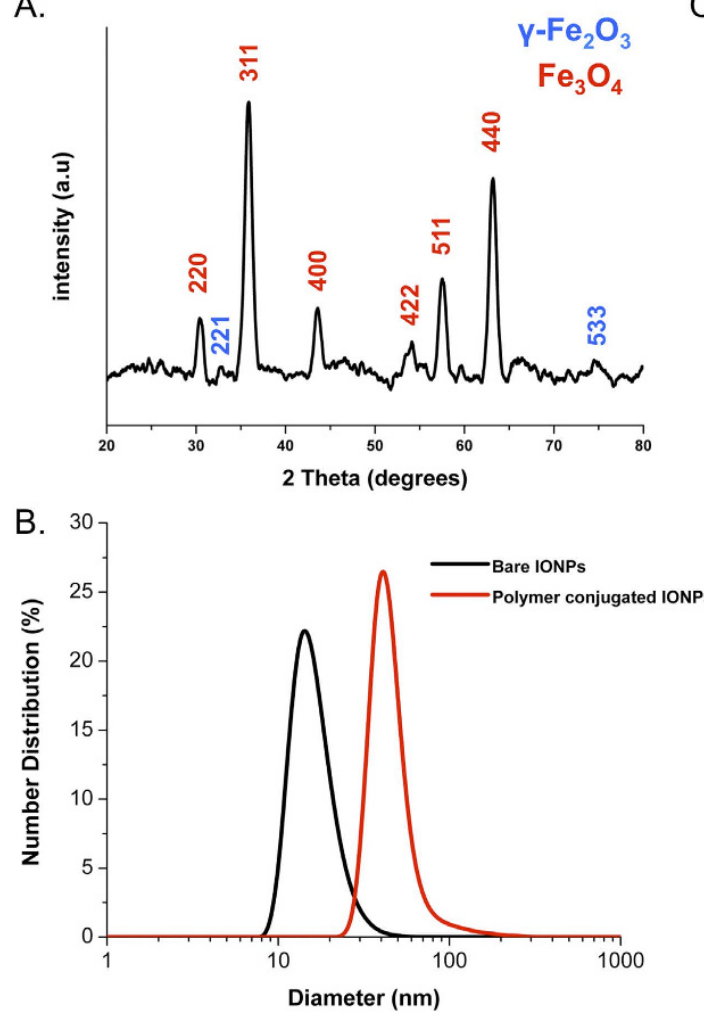

C.

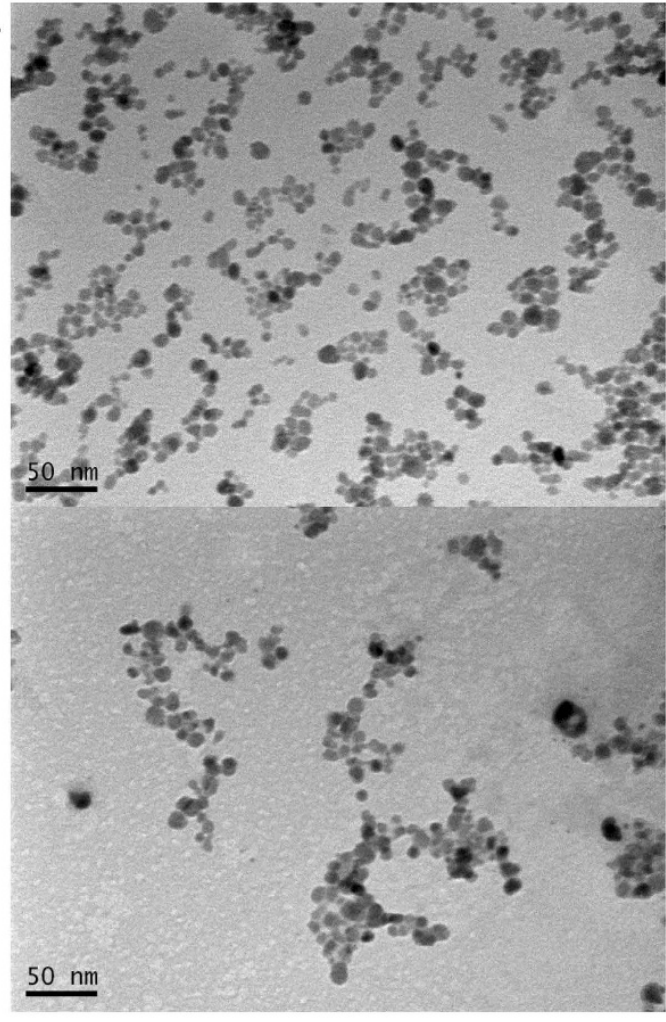

Figure 3. Characterization of POEGA-b-PMAEP@IONPs. (A) Typical X-ray diffraction (XRD) patterns of bare IONPs. Peaks were indexed according to the $F d 3 m$ space group. (B) Number distribution obtained by dynamic light scattering (DLS) of nanoparticles before and after conjugation with polymer. (C) Transmission electron microscopy (TEM) micrographs of dried nanoparticles before (top) and after (bottom) conjugation with polymer.

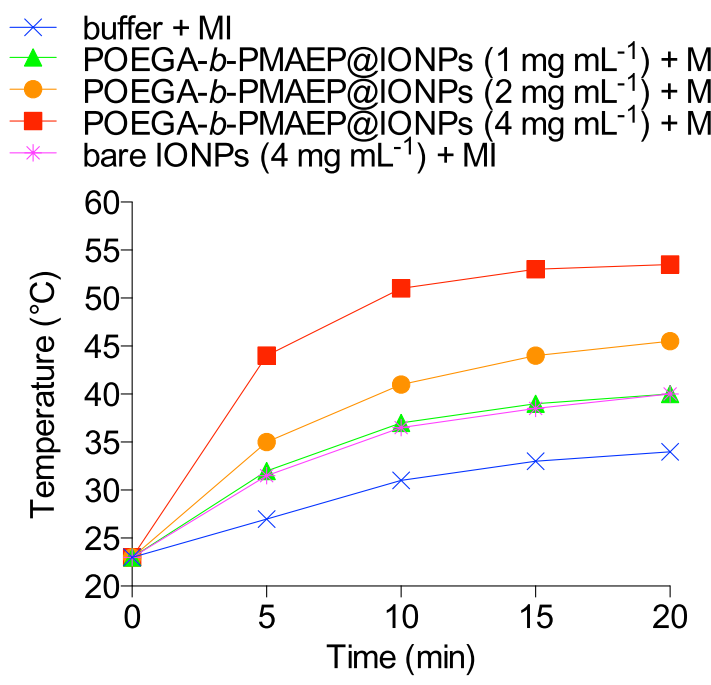

Figure 4. Temperature increases of buffer solutions treated or not with bare IONPs (without polymer) or POEGA-b-PMAEP@IONPs and exposed to an alternating magnetic field $(6.5 \mathrm{~T}, 196 \mathrm{kHz})$ generated with the induction heating system. MI, magnetic induction.

IONPs appeared to rapidly aggregate, while POEGA- $b$-PMAEP@IONPs remained well dispersed (data not shown). The non-aggregative behaviour of the polymer-grafted IONPs appeared to increase their heating capacity, as a solution of only $1 \mathrm{mg} \mathrm{mL}^{-1}$ POEGA- $b$-PMAEP@IONPs was sufficient to reach $40^{\circ} \mathrm{C}$, while a concentration of $4 \mathrm{mg} \mathrm{mL}^{-1}$ of bare IONPs was needed to achieve the same temperature (Fig. 4 ). When the POEGA- $b$-PMAEP@ IONPs concentrations were increased to 2 and $4 \mathrm{mg} \mathrm{mL}^{-1}$, the temperatures went up to 46 and $54^{\circ} \mathrm{C}$, respectively. 
POEGA- $b$-PMAEP@IONPs were used at a final concentration of $1 \mathrm{mg} \mathrm{mL}^{-1}$ (iron-based) for all further hyperthermia experiments.

IONP-induced hyperthermia triggers biofilm dispersal. To test the effect of IONP-mediated hyperthermia on biofilm dispersal, biofilms were grown statically in $14 \mathrm{~mL}$ tubes at room temperature, a set up that is compatible with the coil of the induction heating system used to activate IONPs. When the biofilms were exposed to magnetic induction only, which increases the temperature from $23^{\circ} \mathrm{C}$ to $34^{\circ} \mathrm{C}$, the biofilm biomass was reduced by $20.0 \%$ compared to untreated controls (Fig. 5A). Treatments with POEGA-b-PMAEP@IONPs without induction also induced a $19.7 \%$ decrease in biofilms which may be due to the presence of iron in the nanoparticles. When POEGA- $b$-PMAEP@IONPs were induced with a magnetic field, which leads to an increase in temperature from $23^{\circ} \mathrm{C}$ to $40^{\circ} \mathrm{C}, 69.2 \%$ of the biofilm biomass had dispersed after $20 \mathrm{~min}$ (Fig. 5A,B). Confocal microscopy analysis of biofilms before and after treatment with magnetically induced POEGA- $b$-PMAEP@IONPs confirmed that biofilm biomass was greatly reduced after $20 \mathrm{~min}$ (Fig. 5C). In addition the treatment did not appear to be toxic to bacteria as cells remaining on the surface stained green indicating they were viable bacteria.

Next, the impact of IONPs and IONP-induced hyperthermia on the viability of bacteria was assessed by using colony-forming units (CFU) and intracellular adenosine triphosphate (ATP) analyses. The data show that all treatments resulted in a concomitant increase in planktonic CFU counts and decrease in biofilm CFU counts (Fig. 5D,E), which further confirms that the observed effect are due to a non-toxic dispersal response rather than biofilm reduction via a bactericidal mechanism. Heat induced by magnetic induction only, in the absence of IONP, resulted in a $63.3 \%$ decrease in biofilm CFU and $69.8 \%$ increase in planktonic CFU compared to untreated controls. Treatment with POEGA- $b$-PMAEP@IONPs whether for $20 \mathrm{~min}$ after the initial $24 \mathrm{~h}$ of growth, or from the beginning of growth for the entire $24 \mathrm{~h}$ incubation did not show any toxicity towards planktonic bacteria and in fact these treatments increased planktonic CFU by $116 \%$ and $352 \%$, respectively. The treatment that was most effective at inducing dispersal was when POEGA- $b$-PMAEP@IONPs were added from the start of growth and after $24 \mathrm{~h}$ magnetically activated for $20 \mathrm{~min}$, which resulted in a $93.8 \%$ reduction in biofilm CFU and $614 \%$ increase in planktonic CFU compared to untreated cultures. The trends observed with biofilm CFU analyses were confirmed when performing luciferase-based intracellular ATP measurements of biofilm cells. All treatments resulted in decreased ATP levels, with POEGA- $b$-PMAEP@IONPs added either after or before the $24 \mathrm{~h}$ growth incubation, and magnetically activated for $20 \mathrm{~min}$, resulting in $84.6 \%$ and $81.1 \%$ decrease in ATP, respectively, compared to untreated biofilms (Fig. 5F).

Mutants affected in the c-di-GMP signalling pathway show impaired dispersal in response to hyperthermia. Because temperature-mediated dispersal appeared to involve the secondary messenger c-di-GMP, we then tested the effect of hyperthermia and POEGA- $b$-PMAEP@IONPs on biofilms of two mutant strains affected in lapG and bifA, which are key genes related to c-di-GMP and biofilm dispersal: LapG, as described above is a periplasmic protease that is activated when intracellular c-di-GMP levels decrease, while BifA is a major PDE regulating biofilm development and surface-associated motility in P. aeruginosa ${ }^{35}$. Both lapG and bifA were previously identified in a genetic screen for dispersal deficient mutant biofilms in P. putida ${ }^{36}$. While lapG was shown to not be solely required for temperature-induced dispersal in microfermentor assays, as described above, in the test tube batch biofilm assay, lapG mutant biofilms were only reduced by $7.7 \%$ after magnetic induction alone (representing a temperature increase from $23^{\circ} \mathrm{C}$ to $34^{\circ} \mathrm{C}$ ) versus untreated controls, which represent a $61 \%$ decrease in the dispersal response compared to wild type biofilms (Fig. 6). Similarly bifA mutant biofilms dispersed by only $7.6 \%$ in response to magnetic induction. lap $G$ and bifA biofilms dispersed by $39.8 \%$ and $40.7 \%$ after magnetic induction in the presence of POEGA-b-PMAEP@IONPs, which represent $43 \%$ and $41 \%$ decrease, respectively, in the dispersal response compared to wild type biofilms (Fig. 6).

POEGA-b-PMAEP@IONPs enhance the efficacy of gentamicin antibiotic therapy. Manipulation of biofilm dispersal regulatory pathways is a promising strategy to develop novel measures against antibiotic resistant biofilms, as it allows for the physical removal of biofilm bacteria in a non-toxic manner by stimulating the cells' own genetic circuit for dispersal. Once released from the biofilm, dispersal cells are expected to increase their susceptibility to antimicrobial treatments or immune defences. However the release of bacteria that were once attached may cause downstream problems and lead to sepsis or acute infection. One possible approach to circumvent this problem is to combine dispersal-inducing treatments with adjunctive antimicrobial therapies to inactivate the dispersed cells. Here, to assess the potential of POEGA- $b$-PMAEP@IONPs in combinatorial treatments against biofilms, we tested the effect of POEGA- $b$-PMAEP@IONPs together with the commonly prescribed aminoglycoside antibiotic gentamicin on $P$. aeruginosa biofilms.

In batch biofilm cultures that were treated with gentamicin alone for $20 \mathrm{~min}$, the planktonic phase contained $3.2 \times 10^{7} \mathrm{CFU} \mathrm{mL}{ }^{-1}$, compared to $1.3 \times 10^{8} \mathrm{CFU} \mathrm{mL}^{-1}$ in untreated cultures (Fig. 7A). Treatments with activated POEGA- $b$-PMAEP@IONPs alone induced an increase to $4.3 \times 10^{8}$ planktonic $\mathrm{CFU} \mathrm{mL} \mathrm{mL}^{-1}$, but when combined with gentamicin, the planktonic population was reduced to $1.4 \times 10^{6} \mathrm{CFU} \mathrm{mL}^{-1}$ after $20 \mathrm{~min}$, which represent a 2-log decrease in CFU compared to untreated samples and a 3.2-fold improvement in the efficacy of gentamicin. Further, when the cultures were treated for $2 \mathrm{~h}$ with combined POEGA- $b$-PMAEP@IONPs and gentamicin, planktonic CFU were decreased to $3.6 \times 10^{3} \mathrm{CFU} \mathrm{mL}^{-1}$, thus $\mathrm{a} \geq 4$-log decrease compared to untreated samples (Supplementary information, Fig. S5).

Combined POEGA- $b$-PMAEP@IONPs and gentamicin treatments appeared to be also effective against $P$. aeruginosa biofilms, suggesting that biofilm bacteria that remained on the surface after treatment displayed increased susceptibility towards antimicrobials compared to intact biofilms. Exposure to activated POEGA-b-PMAEP@IONPs alone and gentamicin alone for 20 min resulted in $3.2 \times 10^{6}$ biofilm $\mathrm{CFU} \mathrm{cm}{ }^{-2}$ and $5.3 \times 10^{6}$ biofilm CFU cm${ }^{-2}$,

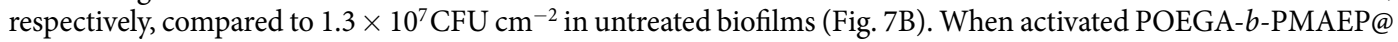


A.

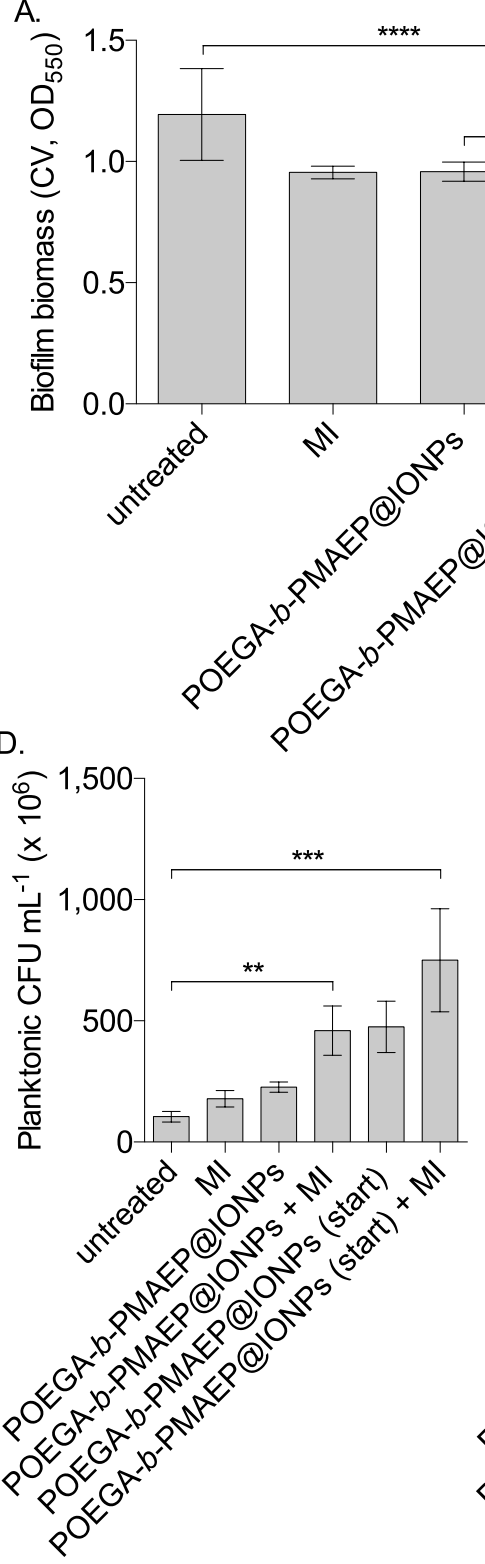

B.

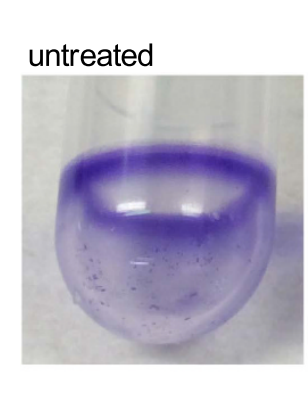

POEGA- $b$-PMAEP

@IONPs + MI

C.

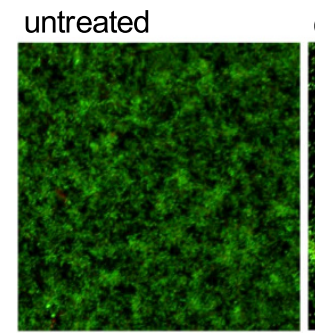

POEGA- $b$-PMAEP @IONPs + MI
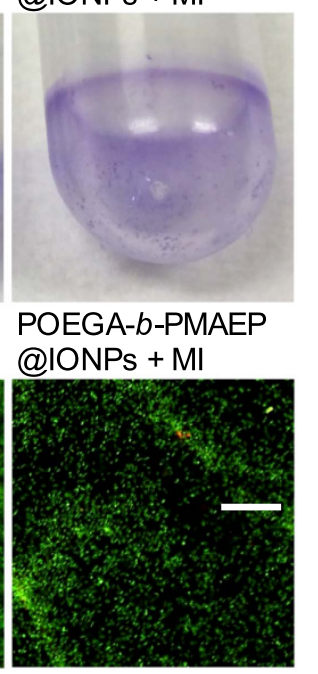

E.

$\mathrm{F}$.

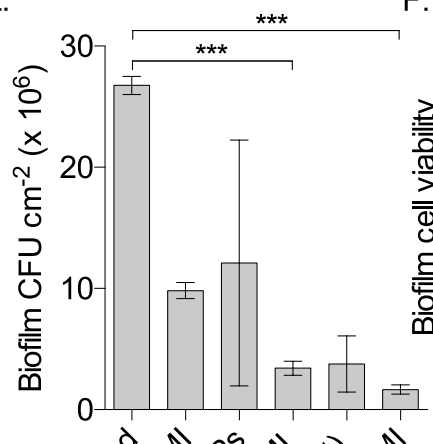

F. 50

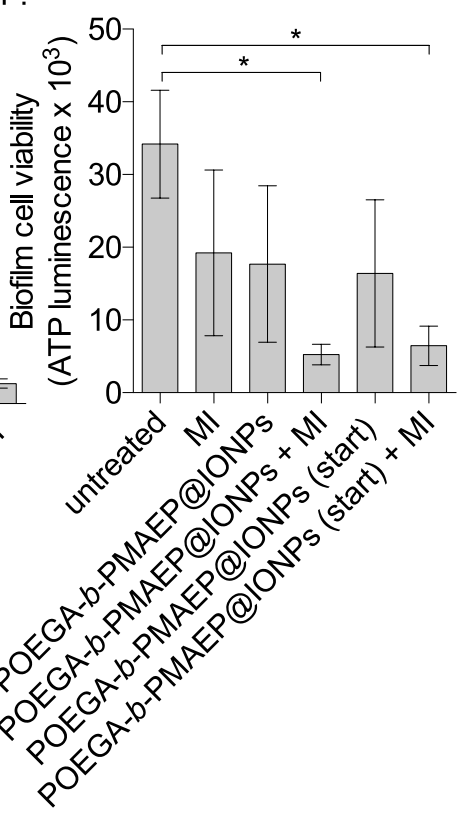

Figure 5. IONP-induced hyperthermia triggers biofilm dispersal in P. aeruginosa. (A) Biofilms were grown for $24 \mathrm{~h}$ in batch culture in test tubes at $23^{\circ} \mathrm{C}$ (room temperature). Then, POEGA- $b$-PMAEP@IONPs $\left(1 \mathrm{mg} \mathrm{mL}^{-1}\right)$ were added to the cultures and the tubes were immediately exposed $(+\mathrm{MI})$ or not to an alternating magnetic field $(6.5 \mathrm{~T}, 196 \mathrm{kHz})$ for a further $20 \mathrm{~min}$ before analysing biofilms by crystal violet (CV) staining. (B) Images of CV stained untreated and treated biofilms. (C) Confocal microscopy images of untreated and treated biofilms stained with LIVE/DEAD (live cells appear green, dead cells appear red; bar $=50 \mu \mathrm{m}$ ). (D-F) After hyperthermia the number of viable cells decreases in the biofilm, but increases in the planktonic phase. Biofilms were grown in test tubes as described above and were treated with POEGA-b-PMAEP@IONPs $\left(1 \mathrm{mg} \mathrm{mL}^{-1}\right)$ either after $24 \mathrm{~h}$, or from the beginning of growth (start). All induced biofilms were exposed to an alternating magnetic field for $20 \mathrm{~min}$ only after the initial $24 \mathrm{~h}$ phase of growth. After that time, bacterial viability was assessed by performing colony-forming units (CFU) analyses of the planktonic (D) and biofilm (E) phases, as well as luciferase-based intracellular ATP measurement of biofilm cells (F). MI, magnetic induction. Error bars represent standard errors $((\mathbf{A}), \mathrm{n} \geq 4 ;(\mathbf{D}-\mathbf{F}), \mathrm{n}=3)$. Asterisks indicate statistically significant difference of treatment versus untreated culture $\left({ }^{\star} P<0.1 ;{ }^{\star *} P<0.01{ }^{* * *} P<0.001 ;{ }^{* * *} P<0.0001\right)$.

IONPs and gentamicin were added together, biofilm bacteria were reduced to $2.9 \times 10^{5} \mathrm{CFU} \mathrm{cm}^{-2}$, almost a $2-\log$ decrease compared to untreated biofilms. Treatments with POEGA- $b$-PMAEP@IONPs and gentamicin in the presence of a magnetic field were significantly $(P<0.0001)$ more efficient in reducing biofilm CFU compared to biofilms that were treated with POEGA-b-PMAEP@IONPs and gentamicin but not activated with a magnetic field. Taken together these data strongly suggest that inducing hyperthermia with polymer-stabilized IONPs and adjunct antimicrobial therapy is a promising strategy to control biofilms and biofilm-related infections. 


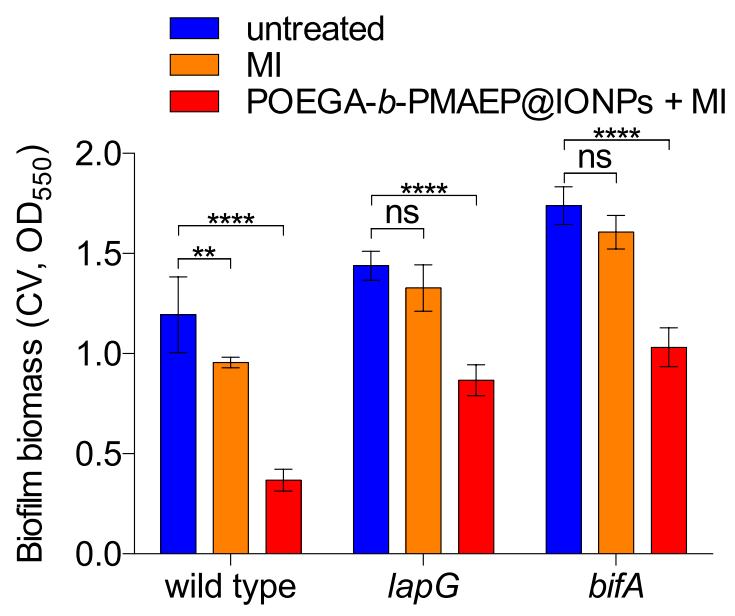

Figure 6. P. aeruginosa strains mutated in key c-di-GMP genes show an attenuated response to IONPinduced hyperthermia. Biofilms of $P$. aeruginosa wild type, lap $G$ (c-di-GMP responsive periplasmic protease) and bifA (c-di-GMP phosphodiesterase) mutant strains were grown in test tubes for $24 \mathrm{~h}$, before being treated with POEGA- $b$-PMAEP@IONPs $\left(1 \mathrm{mg} \mathrm{mL}^{-1}\right)$ and exposed to an alternating magnetic field for $20 \mathrm{~min}$, and finally analysed by crystal violet (CV) staining as described before. MI, magnetic induction. Error bars represent standard errors $(n=3)$. Asterisks indicate statistically significant difference of treatment versus untreated culture $\left({ }^{\star *} P<0.01 ;{ }^{* * *} P<0.0001\right.$; ns, not significant).

\section{Discussion}

The dispersal phase plays critical ecological and evolutionary roles for biofilm populations as it allows for colonization of new habitats and ensures continuity of the species ${ }^{7}$. In this study we reveal that variation in ambient temperature is an important factor regulating dispersal. Temperature is a major determinant for bacterial growth, and the ability to trigger dispersal in response to changes in environmental conditions is likely to optimize chances of survival and successful colonization of new surfaces by dispersal cells. The effect of temperature on biofilm dispersal may not be restricted to $P$. aeruginosa but be conserved across a range of species. In eukaryotes, temperature is well known to be a key factor regulating adaptive physiological changes, for instance by controlling seed germination in plants ${ }^{37}$ and life cycle transitions (e.g. metamorphosis) in animals ${ }^{18}$. Intriguingly, mistletoe plants were recently reported to produce endogenous heat specifically to induce the release and dispersal of seeds from ripe fruits ${ }^{38}$, a process reminiscent of the release of single cells from mature biofilms, which has been referred to as seeding dispersal ${ }^{7}$. Temperature-induced dispersal may also be important in host-microbe interactions and immune responses to pathogen infections, where increasing tissue temperature, e.g. by triggering fever, could contribute to clearing bacterial infections by inducing detachment events and preventing the formation of a persistent biofilm.

Our data suggest that temperature upshifts trigger a signalling pathway involving the secondary messenger c-di-GMP in $P$. aeruginosa. Several reports in the literature have already identified a link between temperature and c-di-GMP, whereby higher temperatures were associated with decreased c-di-GMP levels and biofilm formation in Y. pestis and E. coli $i^{20,21}$, or whereby temperature downshifts were found to trigger biofilm formation in $V$. cholerae $^{22}$. These studies are consistent with the observations reported here that temperature negatively regulates biofilm formation, suggesting that these effects may be conserved across species. The signalling pathway from temperature sensing to dispersal remains to be fully elucidated. The periplasmic protease LapG appears to play a role in regulating dispersal in batch cultures although the transposon mutant strain was not fully impaired in the dispersal response (Fig. 6), suggesting that other mechanisms may be involved in inducing surface detachment. Furthermore, the lap $G$ mutant was not strongly affected in dispersal in continuous flow cultures (Supplementary information, Fig. S1), which may be due to differential expression of c-di-GMP associated genes particularly those interacting with LapG and its cognate c-di-GMP sensor LapD ${ }^{39,40}$ in microfermentor-grown biofilms compared to batch culture biofilms. The PDE BifA also seemed to be involved in regulating dispersal in batch biofilm cultures, but the response was not fully inhibited in the mutant strain (Fig. 6), indicating that other mechanisms such as multiple or redundant PDEs are likely to play a role in this system. Regulation of cellular processes by temperature may occur at different levels, either indirectly via changes in metabolic processes, or directly by affecting enzymatic activity of target enzymes, modulating cell membrane fluidity ${ }^{22}$ or via the stimulation of specific thermosensor proteins capable of interacting with DGC or PDE. Several thermosensors have already been characterized in bacteria, including RNA thermometers involved in cold and heat shock responses, such as CspA in E. coli ${ }^{41}$, and the two-component system DesKR in Bacillus subtilis that responds to alterations in membrane fluidity caused by temperature changes ${ }^{42}$.

The discovery of a role for temperature in regulating dispersal opens new avenues for biofilm control. Here we used IONPs for the induction of local hyperthermia. First, IONPs were conjugated to block copolymers that allow for enhanced stability and functionalization. Previously, POEGA-block-poly(dimethylaminoethyl acrylate) IONPs (POEGA-b-PDMAEA@IONPs) were synthesised that did not show any cytotoxicity ${ }^{43}$, here we used MAEP instead of dimethylaminoethyl acrylate to add more phosphonic acid moieties to bind metal oxides and thus improve adhesion and stability of the block copolymers on the IONPs. The new IONP polymers were found to be highly 
A.
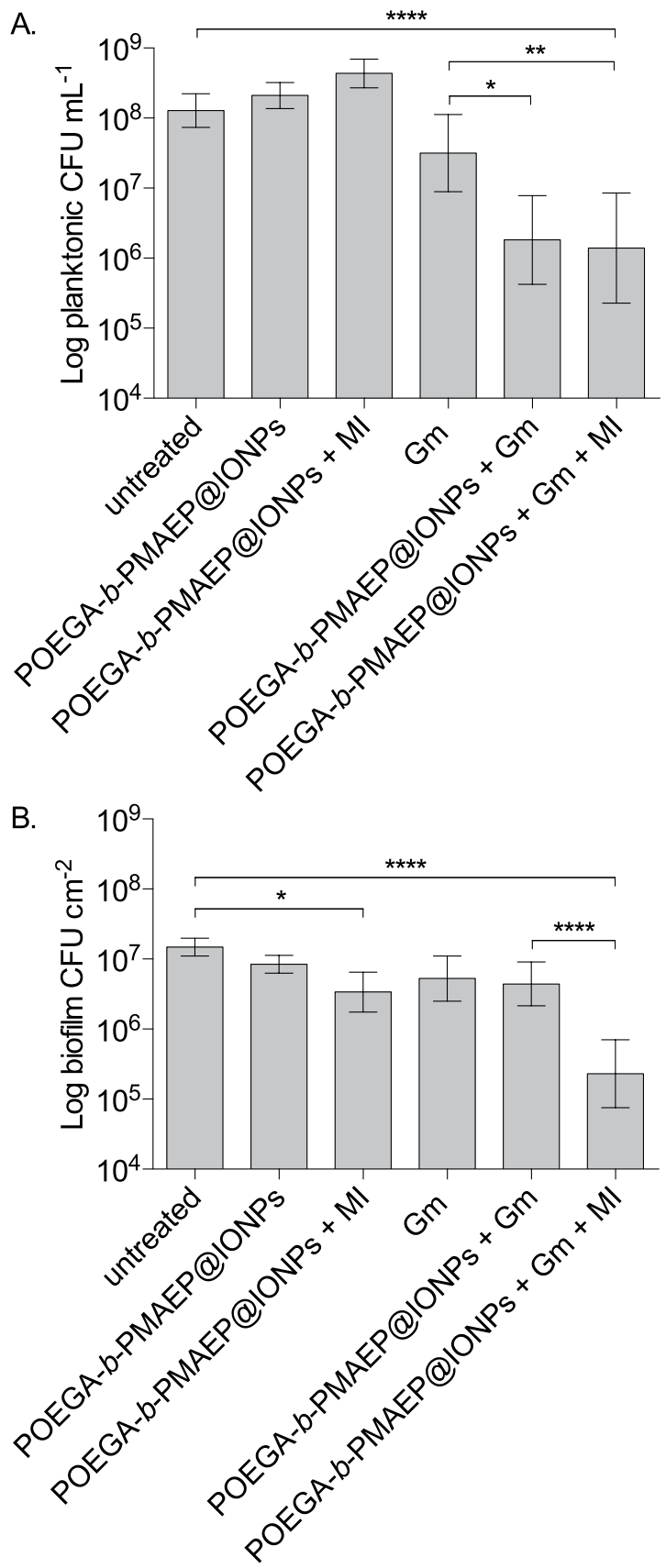

Figure 7. IONP-induced hyperthermia increases the efficacy of the antibiotic gentamicin against both biofilm and planktonic $P$. aeruginosa. Biofilms were grown in test tubes for $24 \mathrm{~h}$ before adding POEGA- $b$ PMAEP@IONPs $\left(1 \mathrm{mg} \mathrm{mL}^{-1}\right)$, gentamicin $\left(\mathrm{Gm}, 2 \mathrm{mg} \mathrm{mL}^{-1}\right)$, a combination of both or no treatment to the cultures, and exposing ( $+\mathrm{MI})$ or not the cultures to an alternating magnetic field $(6.5 \mathrm{~T}, 196 \mathrm{kHz})$ for a further $20 \mathrm{~min}$. After treatment, colony-forming units (CFU) analyses were performed of both the planktonic (A) and biofilm (B) phases. MI, magnetic induction. Error bars represent standard errors $(\mathrm{n} \geq 4)$. Asterisks indicate statistically significant difference of treatment versus untreated culture $\left({ }^{\star} P<0.1 ;{ }^{* *} P<0.01 ;{ }^{* * *} P<0.0001\right)$.

stable and effectively disperse established biofilms. Moreover, both cells remaining on the surface as well as dispersal cells displayed increased susceptibility to gentamicin treatments. Interestingly, enhanced efficacy of a range of antibiotics with different modes of action was already observed in Staphylococcus aureus biofilms when grown at $40^{\circ} \mathrm{C}$ and $45^{\circ} \mathrm{C}$ compared to $35^{\circ} \mathrm{C}^{44}$. This suggests that hyperthermia induced by IONP polymers may also be effective with other antimicrobial agents. In fact, strategies to induce biofilm dispersal, e.g. by using low dose NO donor compound $s^{45}$ or availability of nutrients ${ }^{46}$, have already been shown to restore the sensitivity of biofilm cells towards a range of antimicrobials and antibiotics rather than induce a synergistic effect with one specific antibiotic. IONPs had already been studied for their impact on bacterial biofilms. However, in contrast to all previous studies which investigated a toxic, bactericidal effect of IONPs against planktonic or biofilm bacteria ${ }^{47-50}$, here 
POEGA- $b$-PMAEP@IONPs both in the activated and non-activated states were non-toxic and in fact, as a result of inducing dispersal events, led to an increase in viable planktonic cells (Figs 5D and 7A). Non-toxic therapies to control microbial communities are much preferred, as they are not expected to select for resistant bacterial cells.

In this work, high concentrations of IONPs as well as high magnetic fields were used in order to induce a sufficient increase in temperature. This technology may be directly appropriate for the development of materials with antibiofilm properties that could be remotely activated by applying a magnetic field. For instance, IONP polymers may be applied as surface coatings, for instance via thermal or solvent deposition which would not affect their performance, that would be suitable for the treatment and disinfection of indwelling medical devices such as catheters, or prosthetic implants which are major sources of biofilm-related contaminations in clinical settings ${ }^{51}$. IONPs may also find broad applications in industrial settings, for example for the disinfection of heat exchanger systems, water distribution pipelines or filtration membranes ${ }^{52}$. In the future, new materials may be designed with enhanced functionalities, e.g. targeted release of antibiotics, optimised for administration in humans to treat infectious diseases, and this work may benefit from recent translational research progress to develop IONPs suitable to induce hyperthermia targeted to tumour cells ${ }^{53}$.

\section{Methods}

Bacterial strains, culture media and chemicals. The Gram-negative laboratory strain P. aeruginosa PAO1 was used to characterize the effects of temperature variations on biofilm formation and dispersal. P. aeruginosa PAO1 strains carrying the expression vector pJN105-wspR (iDGC), allowing expression of the DGC WspR under control of the arabinose-inducible $\mathrm{P}_{\text {bad }}$ promoter $\left(\mathrm{Gm}^{\mathrm{R}}\right)$, as well as pJN105 vector control (ctrl) were generously provided by Joana Moscoso/Alain Filloux ${ }^{54}$. P. aeruginosa mutant strains containing a transposon Tn5-derived insertion element $\left(\mathrm{Tc}^{\mathrm{R}}\right)$ in key genes involved in c-di-GMP signalling and biofilm dispersal, bifA (PA4367) and lapG (PA1434), were obtained from the University of Washington P. aeruginosa mutant library: strains PW8371 bifA-D09::ISlacZ/hah and PW3606 lapG-D03::ISlacZ/hah, respectively ${ }^{55}$. Overnight cultures were routinely grown in LB-Miller broth with $10 \mathrm{~g} \mathrm{~L}^{-1} \mathrm{NaCl}$ with shaking at $37^{\circ} \mathrm{C}$. All chemicals were purchased from Ajax and SigmaAldrich, unless otherwise specified. Monomer oligo(ethylene glycol) methyl ether acrylate with an average Mn of $480 \mathrm{~g} \mathrm{~mol}^{-1}$ (OEGA) and monoacryloxy ethyl phosphate (MAEP, Polyscience, Inc.) were used as received. $2,2^{\prime}$-Azobisiobutylronitrile (AIBN) was purified by recrystallization from methanol and stored at $0^{\circ} \mathrm{C}$ until required for further use.

Microfermentor continuous flow biofilm cultures. P. aeruginosa biofilms were grown under continuous flow-through and aerated conditions in glass microfermentors, as previously described ${ }^{26}$ with some slight modifications. The microfermentors received a continuous flow of fresh $\mathrm{M} 9$ minimal medium (containing $48 \mathrm{mM}$ $\mathrm{Na}_{2} \mathrm{HPO}_{4}, 22 \mathrm{mM} \mathrm{KH}_{2} \mathrm{PO}_{4}, 9 \mathrm{mM} \mathrm{NaCl}, 19 \mathrm{mM} \mathrm{NH}_{4} \mathrm{Cl}, 2 \mathrm{mM} \mathrm{MgSO}_{4}, 100 \mu \mathrm{M} \mathrm{CaCl}_{2}, \mathrm{pH} 7.0$ ) with $0.04 \%$ glucose and were immersed in a circulating water bath with a thermostat set at $25^{\circ} \mathrm{C}, 30^{\circ} \mathrm{C}$ or $37^{\circ} \mathrm{C}$. After $24 \mathrm{~h}$ of growth, cells released from the biofilm were monitored by collecting the effluent runoff approximately every $10 \mathrm{~min}$ and measuring the $\mathrm{OD}_{600}$. The $\mathrm{OD}_{600}$ value of the biofilm effluent measured before inducing dispersal was stable and indicated basal, non-specific shedding of bacterial cells from the biofilm. Temperature upshifts were achieved by increasing the thermostat from $25^{\circ} \mathrm{C}$ to $30^{\circ} \mathrm{C}$ or $37^{\circ} \mathrm{C}$, from $30^{\circ} \mathrm{C}$ to $37^{\circ} \mathrm{C}$, or from $37^{\circ} \mathrm{C}$ to $45^{\circ} \mathrm{C}$. For these procedures the temperature in the water bath adjusted within 1 to $3 \mathrm{~min}$. For temperature downshifts, the microfermentors were carefully transferred to another water bath pre-set at $15^{\circ} \mathrm{C}, 25^{\circ} \mathrm{C}$ or $30^{\circ} \mathrm{C}$ without disrupting the medium or airflow in the microfermentors. This procedure ensured minimal physical disruption of the biofilms growing inside the microfermentors.

To test the effect of DGC expression on temperature-induced dispersal, biofilms of $P$. aeruginosa carrying pJN105 and pJN105-wspR plasmids were grown in microfermentors as described above in M9 medium with $0.04 \%$ glucose and $50 \mathrm{\mu g} \mathrm{mL}^{-1}$ gentamicin and without any arabinose for $24 \mathrm{~h}$ at $30^{\circ} \mathrm{C}$. Arabinose was added to $2 \%$ (w/v) in the biofilm medium reservoir $1 \mathrm{~h}$ before increasing the temperature of the water bath to $37^{\circ} \mathrm{C}$. To validate and test the lap $G$ mutant that is affected in dispersal to a range of signals and cues, biofilms of $P$. aeruginosa wild type and lap $G$ transposon mutant were grown in microfermentors as described above for $24 \mathrm{~h}$ at $37^{\circ} \mathrm{C}$ before inducing dispersal by suddenly: (i) adding the NO donor sodium nitroprusside ( $1 \mathrm{mM}$ ) to the biofilm medium; (ii) switching the microfermentor aeration from air to $100 \%$ nitrogen $\left(\mathrm{N}_{2}\right)$ gas to induce $\mathrm{O}_{2}$ depletion; or (iii) switching the biofilm medium to M9 medium without carbon source (no glucose) to induce starvation; or were grown for $24 \mathrm{~h}$ at $30^{\circ} \mathrm{C}$ before increasing the temperature to $37^{\circ} \mathrm{C}$.

For these experiments similar results were observed in at least two or three independent assays, as indicated. The $\mathrm{OD}_{600}$ of the biofilm effluent were normalised to the value before inducing dispersal (time $\mathrm{t}=0$ ) for each biofilm, which typically had a value between 0.05 and 0.15 .

Polymer and nanoparticle characterization. NMR spectroscopy. $\quad{ }^{1} \mathrm{H}$ and ${ }^{31} \mathrm{P}$ NMR spectra were recorded using a Bruker Avance $300(300 \mathrm{MHz})$ spectrometer. $\mathrm{CDCl}_{3}$ and $\mathrm{MeOD}$ were used as solvents. All chemical shifts are reported in parts per million (ppm) relative to tetramethylsilane (TMS), referenced to the residual solvent frequencies ${ }^{1} \mathrm{H}$ NMR: $\mathrm{CDCl}_{3}=7.26, \mathrm{MeOD}=3.31 \mathrm{ppm}$. For ${ }^{31} \mathrm{P}$ NMR spectra, ${ }^{31} \mathrm{P}$ resonances were externally referenced to $85 \% \mathrm{H}_{3} \mathrm{PO} 4$ in $\mathrm{D}_{2} \mathrm{O}$ at $0.00 \mathrm{ppm}$. The monomer (i.e. OEGA) conversion was calculated by the following equation, where $I^{5.9 \mathrm{ppm}}$ and $I^{4.2 \mathrm{ppm}}$ correspond to the integral of the vinyl signal from the monomer at $5.9 \mathrm{ppm}$ $\left({ }^{1} \mathrm{H} \mathrm{mol}^{-1}\right)$ and the ester signal from the monomer/polymer at $4.2 \mathrm{ppm}\left({ }^{2} \mathrm{H} \mathrm{mol}^{-1}\right)$, respectively.

$$
\alpha(\text { OEGA Conversion, } \%)=\left[1-\frac{I^{5.9 p p m}}{\frac{I^{4.2 p p m}}{2}}\right] \times 100 \%
$$


The theoretical molecular weight was calculated by the following equation below:

$$
M_{n}(\text { Theoretical })=\left(\left(\frac{[M]_{0}}{[C T A]_{0}}\right) \times \alpha \times M W_{M}\right)+M W_{C T A}
$$

$[M]_{0}$ : Initial monomer concentration

$[C T A]_{0}$ : Inital RAFT agent concentration

$\alpha$ : Conversion of monomer

$M W_{M}$ : Molecular weight of monomer

$M W_{C T A}$ : Molecular weight of the RAFT agent

Size exclusion chromatography (SEC). SEC analyses of polymer samples were performed in $N, N^{\prime}$-dimethylacetamide [DMAc with $0.03 \% \mathrm{w} / \mathrm{v} \mathrm{LiBr}$ and $0.05 \%$ 2,6-di-butyl-4-methylphenol (BHT)] at $50^{\circ} \mathrm{C}$ (flow rate of $1 \mathrm{~mL} \mathrm{~min}^{-1}$ ) with a Shimadzu modular system comprising and SIL-10AD automatic injector, a Polymer Laboratories $5.0 \mu \mathrm{L}$ bead-size guard column $(50 \times 7.8 \mathrm{~mm})$ followed by four linear PL (Styragel) columns $(105$, 104, 103 and $500 \AA$ ) and an RID-10A differential refractive-index detector. The SEC calibration was performed with narrow-polydispersity polystyrene standards ranging between 104 and 2,000,000 $\mathrm{g} \mathrm{mol}^{-1}$. Polymer solutions at $2-3 \mathrm{mg} \mathrm{mL}^{-1}$ were prepared in the eluent and filtered through $0.45 \mu \mathrm{m}$ filter prior to injection.

Dynamic light scattering (DLS). DLS measurements were performed using a Malvern Zetasizer Nano Series running DTS software ( $4 \mathrm{~mW}$, He-Ne laser, $\lambda=633 \mathrm{~nm}$ ) and an avalanche photodiode (APD) detector. The scattered light was measured at an angle of $175^{\circ}$ for DLS measurements. The temperature was stabilized to $\pm 0.1^{\circ} \mathrm{C}$ of the set temperature. All samples were prepared in Milli-Q water at the concentration of $\sim 0.2 \mathrm{mg} \mathrm{mL}^{-1}$ of polymer and filtered through a $0.45 \mu \mathrm{m}$ pore size filter to remove dust prior to measurement.

Transmission electron microscopy (TEM). Nanoparticles size and morphologies were measured and analysed using a JEOL 1400 transmission electron microscope at an accelerating voltage of $80 \mathrm{kV}$. A drop of samples solution was deposited onto a formvar-coated copper grid and the water was evaporated under air. No staining was applied.

Thermogravimetric analysis (TGA). TGA of IONPs was performed on a Perkin-Elmer Thermogravimetric Analyzer (Pyrus 1 TGA). Pre-dried samples were heated from room temperature to $150^{\circ} \mathrm{C}$ at a constant rate of $20^{\circ} \mathrm{C} \mathrm{min}{ }^{-1}$ using air as the furnace gas. The temperature of $100^{\circ} \mathrm{C}$ was kept constant for 30 min to remove moisture from samples. Then, the temperature was returned to $25^{\circ} \mathrm{C}$ and heated again to $800^{\circ} \mathrm{C}$ at a rate of $20^{\circ} \mathrm{C} \mathrm{min}{ }^{-1}$. The weight loss was calculated through the difference between the weights at room temperature and at $800^{\circ} \mathrm{C}$. The grafting density was estimated using the weight loss (loss-wt) and the surface area of IONPs determined by BET according to the following equation:

$$
\text { Grafting Density }\left(n m^{-2}\right)=\frac{\left(\frac{\text { loss }-w t}{M n_{\text {polymer }}}\right) \times N_{a}}{m_{N P} \times S_{N P}}
$$

$\mathrm{Mn}_{\text {polymer }}$ corresponds to the weight of polymer grafted. $N_{\mathrm{a}}$ is Avogadro's number and $m_{N P}$ is the mass of polymer-grafted iron oxide nanoparticles used for the TGA analysis (i.e. mass of nanoparticles $=$ initial mass before TGA analysis - loss of weight).

$X$-ray diffraction (XRD). The crystal characterization of IONPs was performed using a Philips PANanalytical Xpert X-ray Multipurpose Diffraction System at $40 \mathrm{~mA}$ and $45 \mathrm{kV}$ employing monochromated Cu-Ka radiation $(\lambda=1.541 \AA$, step size $=0.01,0.02$ or 0.05 , time per step $=10$ or $20 \mathrm{~s} / \mathrm{step})$.

POEGA-b-PMAEP@IONPs synthesis. Synthesis of block copolymers POEGA-b-PMAEP. The RAFT agent, 2-(((butylsulfanyl)carbonothioyl)sulfanyl)propanoic acid (BTPA) was prepared according to Ferguson et al. ${ }^{56}$. OEGA (10.08 g, $0.021 \mathrm{~mol})$, BTPA $(0.1925 \mathrm{~g}, 0.0008 \mathrm{~mol})$ and AIBN $\left(0.0265 \mathrm{~g}, 1.6 \times 10^{-4} \mathrm{~mol}\right)$ were dissolved in $25 \mathrm{~mL}$ of toluene in a round bottom flask equipped with a magnetic stirrer bar. The flask was sealed with a rubber septum and the reaction mixture was degassed with $\mathrm{N}_{2}$ gas under ice for $30 \mathrm{~min}$. Then, the degassed solution was placed in a preheated oil bath at $70^{\circ} \mathrm{C}$ and the polymerization was carried out for $4 \mathrm{~h}$. The reaction was terminated by quenching the sample in an ice bath for $15 \mathrm{~min}$ and an aliquot was taken for NMR analysis. The conversion of monomer during the course of polymerization was determined using ${ }^{1} \mathrm{H}$ NMR. The POEGA polymer was purified by precipitating with excess mixture of petroleum ether and diethyl ether $(70: 30, \mathrm{v} / \mathrm{v})$ followed by centrifugation (7,500 rpm for $5 \mathrm{~min})$. The precipitation and centrifugation steps were repeated up to four times to remove any traces of unreacted monomer, and the polymer was dried in a vacuum oven $\left(40^{\circ} \mathrm{C}\right)$ overnight to remove the remaining solvent. The purified POEGA was characterized by ${ }^{1} \mathrm{H}$ NMR and SEC, and stored at $4{ }^{\circ} \mathrm{C}$ until required for further chain extension.

POEGA was used as a macro-RAFT agent for chain extension with MAEP to introduce the functional phosphonic acid group that allows the conjugation to IONPs. POEGA $\mathrm{Mn}=10,000 \mathrm{~g} \mathrm{mol-1}\left(1.0 \mathrm{~g}, 1 \times 10^{-4} \mathrm{~mol}\right)$, MAEP $\left(0.1961 \mathrm{~g}, 1 \times 10^{-3} \mathrm{~mol}\right)$ and AIBN $\left(0.0016 \mathrm{~g}, 1 \times 10^{-5} \mathrm{~mol}\right)$ were dissolved in methanol $(3.5 \mathrm{~mL})$ in a cospak bottle, equipped with a magnetic stirrer bar. The reaction mixture was degassed with $\mathrm{N}_{2}$ gas for $30 \mathrm{~min}$ in an ice bath. The polymerization was carried out in a preheated oil bath at $70^{\circ} \mathrm{C}$ overnight. The reaction was terminated by placing the sample in an ice bath for $15 \mathrm{~min}$ and an aliquot was taken for NMR analysis. The conversion of monomer 
during the course of polymerization was determined using ${ }^{1} \mathrm{H}$ NMR. The copolymer was purified by dialysis against methanol for $48 \mathrm{~h}$ to remove unreacted MAEP, and the purified polymer was then dried in a vacuum oven overnight to remove remaining solvent. The purified copolymer POEGA- $b$-PMAEP was characterized by ${ }^{1} \mathrm{H} N M R$, ${ }^{31} \mathrm{P}$ NMR and SEC, before being stored at $4{ }^{\circ} \mathrm{C}$ until required for further use.

Synthesis of IONPs. IONPs were prepared via chemical co-precipitation method adapted from Jain et al. ${ }^{33}$ with slight modifications. Firstly, $80 \mathrm{~mL}$ of $2 \mathrm{M} \mathrm{FeCl}_{3} \cdot 6 \mathrm{H}_{2} \mathrm{O}(0.16 \mathrm{~mol}, 43.2 \mathrm{~g})$ in $1 \mathrm{M} \mathrm{HCl}$ and $40 \mathrm{~mL}$ of $2 \mathrm{M} \mathrm{FeCl}_{2} \cdot 4 \mathrm{H}_{2} \mathrm{O}$ $(0.08 \mathrm{~mol}, 15.9 \mathrm{~g})$ in $1 \mathrm{M} \mathrm{HCl}$ were mixed in a $2 \mathrm{~L}$ beaker, and the mixture diluted to $1.2 \mathrm{~L}$ deionized water. A total of $250 \mathrm{~mL}$ of ammonium hydroxide $(28 \%)$ was quickly added into the solution of iron chloride, and the mixture was vigorously stirred for $30 \mathrm{~min}$. The colour of the solution immediately changed to black due to the formation of magnetite. The precipitate was collected using a magnet, the supernatant was discarded and the black solid was re-dispersed in a volume of $293 \mathrm{~mL} \mathrm{HNO}_{3}(2 \mathrm{M})$ by stirring for $5 \mathrm{~min}$. Then, an equal volume (i.e. $293 \mathrm{~mL}$ ) of $\mathrm{Fe}\left(\mathrm{NO}_{3}\right)_{3} \cdot 9 \mathrm{H}_{2} \mathrm{O}(0.35 \mathrm{M})$ was added to the magnetite dispersion, followed by heating at $90^{\circ} \mathrm{C}$ for about $1 \mathrm{~h}$ to form maghemite. The precipitate was magnetically collected and then washed twice with $195 \mathrm{~mL} \mathrm{HNO}_{3}(2 \mathrm{M})$ and twice with $250 \mathrm{~mL}$ acetone. Finally, the nanoparticles were dispersed and stored in deionized water which $\mathrm{pH}$ was adjusted to about 1.5-2, resulting in a highly stable dispersion. The synthesized magnetic nanoparticles were characterized by several techniques, including XRD, TGA, TEM and DSL.

Conjugation of block copolymers POEGA-b-PMAEP to IONPs using "grafting onto" approach. Grafting of phosphonic acid bearing copolymer POEGA- $b$-PMAEP on the surface of IONPs was performed as previously described by our group ${ }^{57}$ with some modifications. Briefly, $10 \mathrm{mg}$ IONPs were dispersed in $4 \mathrm{~mL}$ DMSO and the solution was sonicated for $5 \mathrm{~min}$. A solution of $0.0075 \mathrm{mmol}$ of phosphonic acid bearing polymer in $1 \mathrm{~mL}$ DMSO was added dropwise to the dispersion of IONPs. The mixture of polymer and IONPs was sonicated for $3 \mathrm{~min}$, followed by incubation at $50^{\circ} \mathrm{C}$ with shaking at $100 \mathrm{rpm}$ for $48 \mathrm{~h}$. Upon completion, the solution was filtered by passing through a $0.45 \mu \mathrm{m}$ pore size filter to remove the unstabilized nanoparticles and then purified by centrifugation (using the Eppendorf Centrifuge 5804) at 10,000 rpm for $30 \mathrm{~min}$. The functionalized IONPs were isolated at the base of the centrifuge tube. The supernatant was removed and the particles were re-dispersed in acetonitrile. The washing process was repeated twice to give polymer functionalized IONPs synthesized via "grafting onto" method. The purified hybrid product was characterized by TGA, DLS and TEM techniques for grafting efficiency, size and morphology, respectively.

Heat induction derived from the hybrid polymer-IONPs by exposing to an alternating current (AC) magnetic field. Solutions of bare IONPs and POEGA-b-PMAEP@IONPs prepared in PBS (1 mL) at various relative iron concentrations were added to $14-\mathrm{mL}$ round bottom polypropylene tubes (Greiner). IONPs activation was achieved by using a EasyHeat induction heating system (Ambrell), equipped with a helical coil, $25 \mathrm{~mm}$ internal diameter, $60 \mathrm{~mm}$ long with 8 turns. The IONP-containing tube was placed in the centre of the coil and a glass alcohol thermometer was immersed in the solution for temperature measurement. The optimal IONP heating conditions were obtained after $20 \mathrm{~min}$ incubation to an alternating magnetic field at a frequency of $196 \mathrm{kHz}$, which was within the physiological limit of $200 \mathrm{kHz}$ of the human tolerance threshold ${ }^{58}$, and constant $200 \mathrm{~A}$ intensity, which generates a magnetic field of about $6.5 \mathrm{~T}$ in the centre of the coil.

Biofilm dispersal with IONP polymers and eradication with antibiotic-IONP combinatorial treatments. To characterize the effects of IONP polymers on biofilm dispersal, $P$. aeruginos $a$ biofilms were grown at $23^{\circ} \mathrm{C}$ (room temperature) in batch cultures in individual test tubes under static conditions. Briefly in all assays, overnight cultures in LB-Miller broth were diluted to an $\mathrm{OD}_{600}$ of 0.005 in $1 \mathrm{~mL} \mathrm{M9} \mathrm{minimal} \mathrm{medium} \mathrm{with} 0.4 \%$ glucose in $14-\mathrm{mL}$ round bottom polypropylene tubes (Greiner). The tubes were incubated at $23^{\circ} \mathrm{C}$ statically and the biofilms were allowed to grow for $24 \mathrm{~h}$ without any disruption.

IONP polymers were added to the tubes at different concentrations as indicated either after $24 \mathrm{~h}$ incubation or from the beginning of growth. Each treatment was added from a $10 \mu \mathrm{L}$ aliquot of a stock solution at the appropriate concentration of the compound dissolved in PBS. For antibiotic-IONP combinatorial treatments the aminoglycoside gentamicin sulphate was used at a final concentration of $2 \mathrm{mg} \mathrm{mL}^{-1}$ and added to the cultures either alone or together with POEGA- $b$-PMAEP@IONPs. After adding the treatments, the tubes were carefully transferred to a mock location on the bench (no magnetic induction) or to the centre of the coil of the EasyHeat induction heating system operating at constant $200 \mathrm{~A}$ intensity and $196 \mathrm{kHz}$ frequency and incubated there for a further 20 min before quantifying the biomass or viability of both planktonic and biofilm bacteria. For combinatorial treatments with POEGA-b-PMAEP@IONPs and gentamicin, biofilms to which the treatments had been added were exposed to magnetic induction or left at a mock location for either $20 \mathrm{~min}$ or for $2 \mathrm{~h}$.

Biofilm biomass was quantified by using the crystal violet staining method as previously described ${ }^{26}$. Briefly, after incubation the culture supernatant was removed and the biofilm on the tube surfaces was first washed once with PBS $(1 \mathrm{~mL})$, before adding $0.03 \%$ crystal violet stain $(1 \mathrm{~mL})$. The tube was incubated on the bench for $20 \mathrm{~min}$ before removing the dye and washing the internal surfaces twice with PBS. Photographs of the stained biofilms were obtained using a digital camera. The amount of remaining crystal violet-stained biofilm was quantified by adding $100 \%$ ethanol $(1 \mathrm{~mL})$ and measuring the $\mathrm{OD}_{550}$ of the homogenized suspension by using a microtitre plate reader (Wallac Victor ${ }^{2}$, Perkin-Elmer). OD measurements of control tubes where no bacteria were added at the beginning of the experiment were subtracted from all values (i.e. $\mathrm{OD}_{550}=0.10$ ).

To determine bacterial viability, planktonic and biofilm colony-forming units (CFU) analyses were determined by a drop plate method ${ }^{59}$. For planktonic analysis, free-floating cells in the biofilm supernatant were serially diluted in sterile PBS and plated onto LB agar. For biofilm analysis, cells attached on the interior surfaces of the tubes (surface area $3.8 \mathrm{~cm}^{2}$ ) were washed twice with sterile PBS to remove loosely attached bacteria, before being resuspended 
and homogenised in PBS by incubating in a sonication bath $\left(150 \mathrm{~W}\right.$, Unisonics) for $20 \mathrm{~min}^{60}$. Then, cells were serially diluted and plated onto LB agar. CFU were enumerated after $24 \mathrm{~h}$ incubation at $37^{\circ} \mathrm{C}$. The BacTiter-Glo Microbial Cell Viability Assay (Promega, Alexandria, Australia) was also used, which is based on quantitation of the ATP present in bacteria by using a thermostable luciferase. After the final $20 \mathrm{~min}$ incubation with various treatments and magnetic induction, biofilm cells were resuspended and homogenized in PBS as described above. Resuspended biofilm cells were mixed with BacTiter-Glo reagent and after $5 \mathrm{~min}$ the luminescence was measured by using a multimode microtitre plate reader (Wallac Victor ${ }^{2}$, Perkin-Elmer).

All assays included at minimum 2 replicates and were repeated in at least 2 or 3 independent experiments. Data analysis was performed using means (crystal violet, ATP measurements) or geometric means (CFU) and compared among groups via one-way ANOVA, followed by Tukey's post test for individual comparisons. Differences were deemed statistically significant at a $P$ value $<0.1$.

Confocal microscopy analysis. For microscopy analysis, narrow glass coverslips $(10.5 \times 35 \mathrm{~mm}$, ProSciTech, Townsville, Australia) were inserted in the biofilm culture tubes and P. aeruginosa biofilms were grown otherwise as described above. After $24 \mathrm{~h}$ incubation and 20 min treatment, the coverslips harbouring biofilms were carefully removed from the tubes by using sterile tweezers and rinsed twice with PBS before wiping clean one side and staining the other side with LIVE/DEAD BacLight bacterial viability kit reagents (Molecular Probes) according to the manufacturer's procedure. Three hundred microliters of the staining solution (1:1,000 dilution of each SYTO9 and propidium iodide components in PBS) was trapped between the biofilm sample on the coverslip and a standard microscope slide. After 20 min incubation at room temperature in the dark, the samples were observed with an Olympus FV1000 Confocal Inverted Microscope, and imaged with Leica DFC 480 camera. Cells that were stained green were considered to be viable, those that stained red were considered to be dead.

\section{References}

1. Poole, K. Bacterial stress responses as determinants of antimicrobial resistance. J. Antimicrob. Chemother. 67, 2069-2089 (2012).

2. Hoiby, N., Bjarnsholt, T., Givskov, M., Molin, S. \& Ciofu, O. Antibiotic resistance of bacterial biofilms. Int. J. Antimicrob. Agents 35, 322-332 (2010).

3. Lebeaux, D., Ghigo, J. M. \& Beloin, C. Biofilm-related infections: bridging the gap between clinical management and fundamental aspects of recalcitrance toward antibiotics. Microbiol. Mol. Biol. Rev. 78, 510-543 (2014).

4. Flemming, H.-C. in Biofilm Highlights Vol. 5 Springer Series on Biofilms (eds Hans-Curt Flemming, Jost Wingender \& Ulrich Szewzyk) Ch. 5, 81-109 (Springer Berlin Heidelberg, 2011).

5. Chaves Simões, L. \& Simões, M. Biofilms in drinking water: problems and solutions. RSC Adv. 3, 2520-2533 (2013).

6. Christensen, L. D. et al. Clearance of Pseudomonas aeruginosa foreign-body biofilm infections through reduction of the cyclic diGMP level in the bacteria. Infect. Immun. 81, 2705-2713 (2013).

7. McDougald, D., Rice, S. A., Barraud, N., Steinberg, P. D. \& Kjelleberg, S. Should we stay or should we go: mechanisms and ecological consequences for biofilm dispersal. Nat. Rev. Microbiol. 10,39-50 (2012).

8. Sauer, K. et al. Characterization of nutrient-induced dispersion in Pseudomonas aeruginosa PAO1 biofilm. J. Bacteriol. 186, 7312-7326 (2004).

9. Schleheck, D. et al. Pseudomonas aeruginosa PAO1 preferentially grows as aggregates in liquid batch cultures and disperses upon starvation. PLoS One 4, e5513 (2009).

10. Thormann, K. M., Saville, R. M., Shukla, S. \& Spormann, A. M. Induction of rapid detachment in Shewanella oneidensis MR-1 biofilms. J. Bacteriol. 187, 1014-1021 (2005).

11. Barraud, N., Kelso, M. J., Rice, S. A. \& Kjelleberg, S. Nitric oxide: a key mediator of biofilm dispersal with applications in infectious diseases. Curr. Pharm. Design 21, 31-42 (2015).

12. Banin, E., Brady, K. M. \& Greenberg, E. P. Chelator-induced dispersal and killing of Pseudomonas aeruginosa cells in a biofilm. Appl. Environ. Microbiol. 72, 2064-2069 (2006).

13. Rice, S. A. et al. Biofilm formation and sloughing in Serratia marcescens are controlled by quorum sensing and nutrient cues. J. Bacteriol. 187, 3477-3485 (2005).

14. Boles, B. R. \& Horswill, A. R. agr-mediated dispersal of Staphylococcus aureus biofilms. PLoS Pathog. 4, e1000052 (2008).

15. Dow, J. M. et al. Biofilm dispersal in Xanthomonas campestris is controlled by cell-cell signaling and is required for full virulence to plants. Proc. Natl. Acad. Sci. USA. 100, 10995-11000 (2003).

16. Davies, D. G. \& Marques, C. N. A fatty acid messenger is responsible for inducing dispersion in microbial biofilms. J. Bacteriol. 191, 1393-1403 (2009).

17. Römling, U., Galperin, M. Y. \& Gomelsky, M. Cyclic di-GMP: the first 25 years of a universal bacterial second messenger. Microbiol. Mol. Biol. Rev. 77, 1-52 (2013).

18. Bosch, T. C. et al. How do environmental factors influence life cycles and development? An experimental framework for earlydiverging metazoans. Bioessays 36, 1185-1194 (2014).

19. Kaplan, J. B. \& Fine, D. H. Biofilm dispersal of Neisseria subflava and other phylogenetically diverse oral bacteria. Appl. Environ. Microbiol. 68, 4943-4950 (2002).

20. Perry, R. D. et al. Temperature regulation of the hemin storage $\left(\mathrm{Hms}^{+}\right)$phenotype of Yersinia pestis is posttranscriptional. J. Bacteriol. 186, 1638-1647 (2004).

21. Nakasone, Y., Ono, T. A., Ishii, A., Masuda, S. \& Terazima, M. Temperature-sensitive reaction of a photosensor protein YcgF: possibility of a role of temperature sensor. Biochemistry 49, 2288-2296 (2010).

22. Townsley, L. \& Yildiz, F. H. Temperature affects c-di-GMP signaling and biofilm formation in Vibrio cholerae. Environ. Microbiol., accepted doi: 10.1111/1462-2920.12799 (2015).

23. Laurent, S. et al. Magnetic iron oxide nanoparticles: synthesis, stabilization, vectorization, physicochemical characterizations, and biological applications. Chem. Rev. 108, 2064-2110 (2008).

24. Boyer, C. et al. The stabilization and bio-functionalization of iron oxide nanoparticles using heterotelechelic polymers. J. Mater. Chem. 19, 111-123 (2009)

25. Boyer, C., Whittaker, M. R., Bulmus, V., Liu, J. \& Davis, T. P. The design and utility of polymer-stabilized iron-oxide nanoparticles for nanomedicine applications. NPG Asia Mater. 2, 23-30 (2010).

26. Barraud, N., Moscoso, J. A., Ghigo, J. M. \& Filloux, A. Methods for studying biofilm dispersal in Pseudomonas aeruginosa. Methods Mol. Biol. 1149, 643-651 (2014).

27. Mikkelsen, H., Hui, K., Barraud, N. \& Filloux, A. The pathogenicity island encoded PvrSR/RcsCB regulatory network controls biofilm formation and dispersal in Pseudomonas aeruginosa PA14. Mol. Microbiol. 89, 450-463 (2013).

28. Gjermansen, M., Nilsson, M., Yang, L. \& Tolker-Nielsen, T. Characterization of starvation-induced dispersion in Pseudomonas putida biofilms: genetic elements and molecular mechanisms. Mol. Microbiol. 75, 815-826 (2010). 
29. Newell, P. D., Boyd, C. D., Sondermann, H. \& O’Toole, G. A. A c-di-GMP effector system controls cell adhesion by inside-out signaling and surface protein cleavage. PLoS Biol. 9, e1000587 (2011).

30. Barraud, N. et al. Nitric oxide signaling in Pseudomonas aeruginosa biofilms mediates phosphodiesterase activity, decreased cyclic di-GMP levels, and enhanced dispersal. J. Bacteriol. 191, 7333-7342 (2009).

31. An, S., Wu, J. \& Zhang, L. H. Modulation of Pseudomonas aeruginosa biofilm dispersal by a cyclic-di-GMP phosphodiesterase with a putative hypoxia-sensing domain. Appl. Environ. Microbiol. 76, 8160-8173 (2010).

32. Massart, R. Preparation of aqueous magnetic liquids in alkaline and acidic media. IEEE Trans. Magn. 17, 1247-1248 (1981).

33. Jain, N., Wang, Y., Jones, S. K., Hawkett, B. S. \& Warr, G. G. Optimized steric stabilization of aqueous ferrofluids and magnetic nanoparticles. Langmuir : the ACS journal of surfaces and colloids 26, 4465-4472 (2010).

34. Torres-Lugo, M. \& Rinaldi, C. Thermal potentiation of chemotherapy by magnetic nanoparticles. Nanomedicine 8, 1689-1707 (2013).

35. Kuchma, S. L. et al. BifA, a cyclic-di-GMP phosphodiesterase, inversely regulates biofilm formation and swarming motility by Pseudomonas aeruginosa PA14. J. Bacteriol. 189, 8165-8178 (2007).

36. López-Sánchez, A., Jiménez-Fernández, A., Calero, P., Gallego, L. D. \& Govantes, F. New methods for the isolation and characterization of biofilm-persistent mutants in Pseudomonas putida. Environ. Microbiol. Rep. 5, 679-685 (2013).

37. Probert, R. J. in Seeds: the ecology of regeneration in plant communities (ed. M. Fenner) Ch. 11, 261-292 (CAB International, 2000).

38. deBruyn, R. A., Paetkau, M., Ross, K. A., Godfrey, D. V. \& Friedman, C. R. Thermogenesis-triggered seed dispersal in dwarf mistletoe. Nat. Commun. 6, 6262 (2015).

39. Cooley, R. B. et al. Cyclic di-GMP-regulated periplasmic proteolysis of a Pseudomonas aeruginosa type Vb secretion system substrate. J. Bacteriol., accepted doi: 10.1128/JB.00369-00315 (2015).

40. Rybtke, M. et al. The LapG protein plays a role in Pseudomonas aeruginosa biofilm formation by controlling the presence of the CdrA adhesin on the cell surface. MicrobiologyOpen, accepted doi: 10.1002/mbo3.301 (2015).

41. Kortmann, J. \& Narberhaus, F. Bacterial RNA thermometers: molecular zippers and switches. Nat. Rev. Microbiol. 10, 255-265 (2012).

42. Aguilar, P. S., Hernandez-Arriaga, A. M., Cybulski, L. E., Erazo, A. C. \& de Mendoza, D. Molecular basis of thermosensing: a twocomponent signal transduction thermometer in Bacillus subtilis. EMBO J. 20, 1681-1691 (2001).

43. Boyer, C. et al. Anti-fouling magnetic nanoparticles for siRNA delivery. J. Mater. Chem. 20, 255-265 (2010).

44. Hajdu, S. et al. Increased temperature enhances the antimicrobial effects of daptomycin, vancomycin, tigecycline, fosfomycin, and cefamandole on staphylococcal biofilms. Antimicrob. Agents Chemother. 54, 4078-4084 (2010).

45. Barraud, N. et al. Involvement of nitric oxide in biofilm dispersal of Pseudomonas aeruginosa. J. Bacteriol. 188, 7344-7353 (2006).

46. Sommerfeld Ross, S. \& Fiegel, J. Nutrient dispersion enhances conventional antibiotic activity against Pseudomonas aeruginosa biofilms. Int. J. Antimicrob. Agents 40, 177-181 (2012).

47. Taylor, E. N. et al. Superparamagnetic iron oxide nanoparticles (SPION) for the treatment of antibiotic-resistant biofilms. Small 8, 3016-3027 (2012).

48. Kim, M. H. et al. Magnetic nanoparticle targeted hyperthermia of cutaneous Staphylococcus aureus infection. Ann. Biomed. Eng. 41, 598-609 (2013).

49. Niemirowicz, K. et al. Gold-functionalized magnetic nanoparticles restrict growth of Pseudomonas aeruginosa. Int. J. Nanomedicine 9, 2217-2224 (2014).

50. Thukkaram, M., Sitaram, S., Kannaiyan, S. K. \& Subbiahdoss, G. Antibacterial efficacy of iron-oxide nanoparticles against biofilms on different biomaterial surfaces. Int. J. Biomater. 2014, 716080 (2014).

51. Lebeaux, D. et al. Management of infections related to totally implantable venous-access ports: challenges and perspectives. Lancet Infect. Dis. 14, 146-159 (2014).

52. Murthy, P. S. \& Venkatesan, R. in Marine and Industrial Biofouling Vol. 4 Springer Series on Biofilms (eds Hans-Curt Flemming, P. Sriyutha Murthy, R. Venkatesan \& Keith Cooksey) Ch. 4, 65-101 (Springer Berlin Heidelberg, 2009).

53. Anselmo, A. \& Mitragotri, S. A review of clinical translation of inorganic nanoparticles. AAPS J., 1-14 (2015).

54. Moscoso, J. A. Regulatory cascades involving cyclic di-GMP signalling in Pseudomonas aeruginosa Ph.D. thesis, Imperial College London (2014).

55. Jacobs, M. A. et al. Comprehensive transposon mutant library of Pseudomonas aeruginosa. Proc. Natl. Acad. Sci. USA. 100, 14339-14344 (2003).

56. Ferguson, C. J. et al. Ab initio emulsion polymerization by RAFT-controlled self-assembly. Macromolecules 38, 2191-2204 (2005).

57. Basuki, J. S. et al. Grafting of $\mathrm{P}(\mathrm{OEGA})$ onto magnetic nanoparticles using $\mathrm{Cu}(0)$ mediated polymerization: comparing grafting "from" and "to" approaches in the search for the optimal material design of nanoparticle MRI contrast agents. Macromolecules 46, 6038-6047 (2013).

58. Hergt, R. \& Dutz, S. Magnetic particle hyperthermia—biophysical limitations of a visionary tumour therapy. J. Magn. Magn. Mater. 311, 187-192 (2007).

59. Hoben, H. J. \& Somasegaran, P. Comparison of the pour, spread, and drop plate methods for enumeration of Rhizobium spp. in inoculants made from presterilized peat. Appl. Environ. Microbiol. 44, 1246-1247 (1982).

60. Barraud, N., Buson, A., Jarolimek, W. \& Rice, S. A. Mannitol enhances antibiotic sensitivity of persister bacteria in Pseudomonas aeruginosa biofilms. PLoS One 8, e84220 (2013).

\section{Acknowledgements}

The authors would like to acknowledge the facilities and the scientific and technical assistance of the Mark Wainwright Analytical Centre at The University of New South Wales (UNSW), as well as Dr. May Lim for help with the magnetic field generator. This work was supported by grants from the Australian Research Council (ARC), the Centre for Marine Bio-Innovation and UNSW. C.B. is supported by ARC Future Fellowship FT120100096 and N.B. by ARC grant DE120101604.

\section{Author Contributions}

C.B. and N.B. designed the project. T.K.N., H.T.T.D., R.S. and N.B. performed the experiments. C.B. and N.B. contributed reagents and materials. T.K.N., H.T.T.D., C.B. and N.B. analysed and interpreted data. T.K.N., C.B. and N.B. wrote the main manuscript text. All authors reviewed the manuscript.

\section{Additional Information}

Supplementary information accompanies this paper at http://www.nature.com/srep

Competing financial interests: The authors declare no competing financial interests.

How to cite this article: Nguyen, T.-K. et al. Iron oxide nanoparticle-mediated hyperthermia stimulates dispersal in bacterial biofilms and enhances antibiotic efficacy. Sci. Rep. 5, 18385; doi: 10.1038/srep18385 (2015). 
(c) (i) This work is licensed under a Creative Commons Attribution 4.0 International License. The images or other third party material in this article are included in the article's Creative Commons license, unless indicated otherwise in the credit line; if the material is not included under the Creative Commons license, users will need to obtain permission from the license holder to reproduce the material. To view a copy of this license, visit http://creativecommons.org/licenses/by/4.0/ 